Document downloaded from:

http://hdl.handle.net/10251/63519

This paper must be cited as:

Sanguesa, JA.; Fogue, M.; Garrido, P.; Martinez, FJ.; Cano Escribá, JC.; Tavares De Araujo Cesariny Calafate, CM.; Manzoni, P. (2015). RTAD: A real-time adaptive dissemination system for VANETs. Computer Communications. 60:53-70. doi:10.1016/j.comcom.2015.01.017.

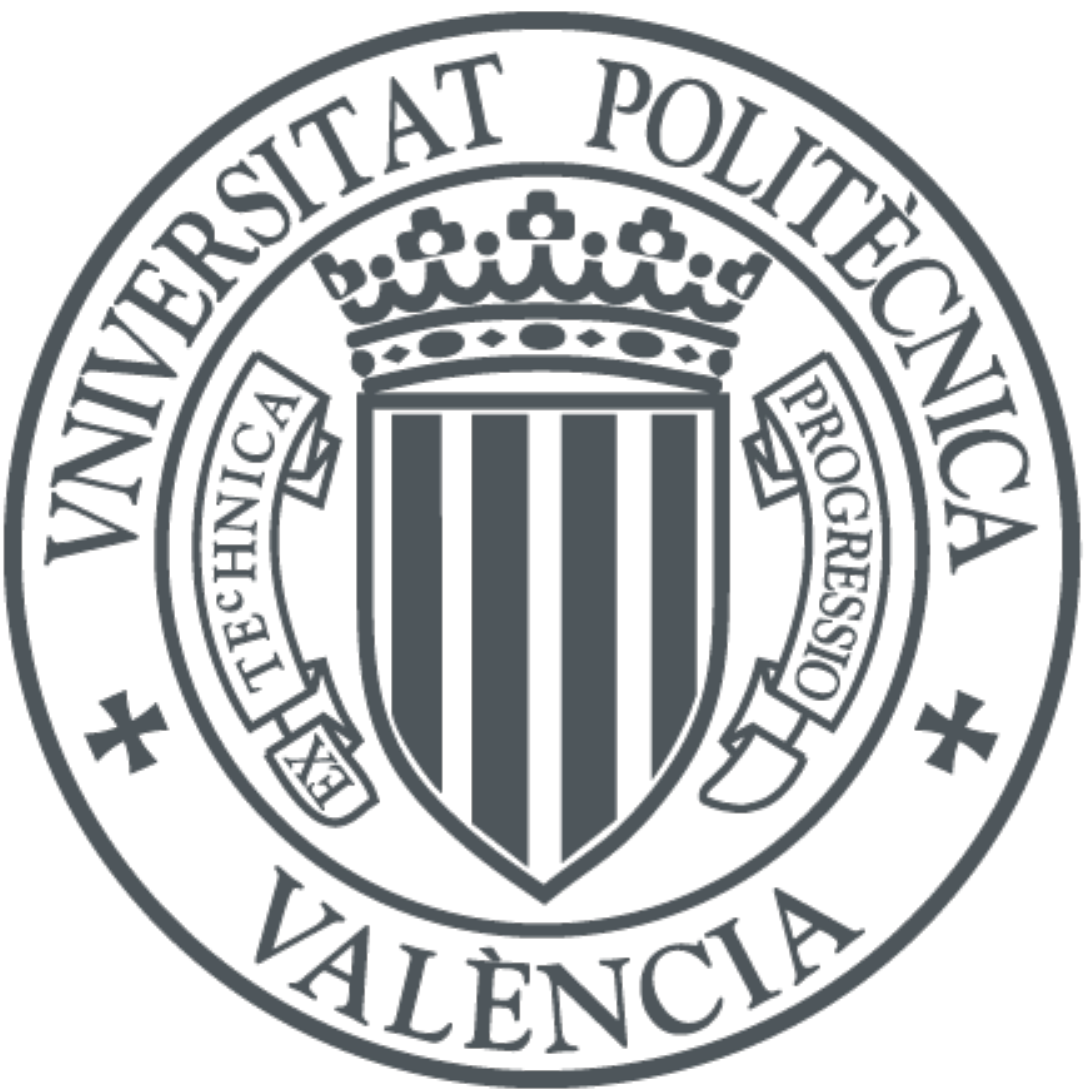

The final publication is available at

http://dx.doi.org/10.1016/j.comcom.2015.01.017

Copyright Elsevier

Additional Information 


\title{
RTAD: a Real-time Adaptive Dissemination System for VANETs
}

Julio A. Sanguesa, Manuel Fogue, Piedad Garrido, Francisco J. Martinez

\author{
University of Zaragoza, Spain \\ Email: \{jsanguesa,mfogue, piedad,f.martinez\}@unizar.es \\ Juan-Carlos Cano, Carlos T. Calafate, Pietro Manzoni \\ Universitat Politècnica de València, Spain \\ Email: \{jucano, calafate,pmanzoni\}@disca.upv.es
}

\begin{abstract}
Efficient message dissemination is of utmost importance to propel the development of useful services and applications in Vehicular ad hoc Networks (VANETs). In this paper, we propose a novel adaptive system that allows each vehicle to automatically adopt the optimal dissemination scheme in order to fit the warning message delivery policy to each specific situation. Our mechanism uses as input parameters the vehicular density and the topological characteristics of the environment where the vehicles are located, in order to decide which dissemination scheme to use. We compare our proposal with respect to two static dissemination schemes (eMDR and NJL), and three adaptive dissemination systems (UV-CAST, FDPD, and DV-CAST). Simulation results demonstrate that our approach significantly improves upon these solutions, being able to support more efficient warning message dissemination in all situations ranging from low densities with complex maps, to high densities in simple scenarios. In particular, RTAD improves existing approaches in terms of percentage of vehicles informed, while significantly reducing the number of messages sent, thus mitigating broadcast storms.
\end{abstract}

Keywords: Vehicular ad hoc networks; warning message dissemination; adaptive systems; VANETs. 


\section{Introduction}

Modern Intelligent Transportation Systems (ITS) are being propelled by the development and adoption of wireless telecommunications and computing technologies, which allow our roads and highways to be both safer and more efficient transportation platforms. Vehicular ad hoc Networks (VANETs) are wireless communication networks which support cooperative driving among vehicles on the road. In such networks, vehicles act as communication nodes and relays, establishing dynamic vehicular networks together with other nearby vehicles (Boukerche et al., 2008).

The specific characteristics of VANETs favor the development of attractive and challenging services and applications, including road safety (Ghandour et al., 2014), traffic flow management (Sanchez-Medina et al., 2010), road status monitoring (Li et al., 2008), environmental protection (Noor et al., 2012), and mobile infotainment (Salvo et al., 2012). In this work we focus on traffic safety and efficient warning message dissemination, where the main goal is to reduce the latency and to increase the accuracy of the information received by nearby vehicles when a dangerous situation occurs (Zhou et al., 2008).

In a VANET, any vehicle detecting an abnormal situation (i.e. accident, slippery road, etc.) rapidly starts notifying the anomaly to nearby vehicles to spread the alert information in a short period of time (Kumar et al., 2014). Thus, broadcasting warning messages can be useful to alert nearby vehicles. However, this dissemination is strongly affected by: (i) the signal attenuation due to the distance between the sender and receiver (especially in low vehicular density areas), (ii) the effect of obstacles in signal transmission (very usual in urban areas, e.g., due to buildings), and (iii) a reduced message delivery effectiveness due to serious redundancy, contention, and massive packet collisions provoked by simultaneous forwarding, usually known as broadcast storm (prone to occur in highly congested areas) (Tseng et al., 2002). Therefore, knowing the density of vehicles and the characteristics of the area where the vehicles are moving (e.g., in terms of topological complexity) can offer better opportunities for message 
delivery.

We consider that new adaptive proposals for warning message dissemination in urban environments are needed, offering efficient broadcasting techniques around the affected area, taking into account the current vehicular density, as well as the topology of the scenario where vehicles are located. This can be beneficial in order to increase the efficiency of the warning message dissemination process, and also to reduce broadcast storm related problems. The objective is to increase the probability of correctly alert surrounding vehicles, thereby preventing new dangerous situations.

In this paper we propose RTAD, a real-time adaptive dissemination system that allows each vehicle to automatically adopt the optimal dissemination scheme to adapt the warning message delivery policy to each specific situation. Our mechanism uses as input parameters the estimated vehicular density (according to a neighbor-based density estimation scheme) and the topological characteristics of the environment where the vehicles are located, using them to decide which dissemination scheme to use. The main goal is to maximize the message delivery effectiveness while generating a reduced number of messages and, thus, avoiding or mitigating broadcast storms. In addition, we also propose the Nearest Junction Located (NJL), our novel warning message dissemination scheme specially designed for being used in highly congested urban areas.

This work is an extended version of a preliminary contribution presented in (Sanguesa et al., 2013b). In particular, we have implemented and assessed the feasibility of RTAD. Additionally, since the RTAD system needs to estimate the vehicle density to select the most appropriate broadcast scheme, our approach uses the number of neighbors, instead of the number of beacons received, to estimate the vehicle density. In order to assess RTAD's performance, we tested it under four different scenarios: two of them previously used to calibrate the algorithm (Amsterdam and Los Angeles), and two new scenarios (Sydney and Santiago de Chile) characterized by larger map areas, as well as having one (Sydney) or two (Santiago de Chile) different downtown areas. Finally, we have included a comparison between our proposal and two static broadcast schemes 
(eMDR and NJL), as well as three adaptive systems (UV-CAST, FPDP and DV-CAST).

The paper is organized as follows: in Section 2 we present the simulation environment used to validate our proposal and some previous concepts. In Section 3 we make a preliminary analysis of different broadcast schemes, and we present the optimal broadcast selection algorithm proposed. Section 4 introduces RTAD, our real-time adaptive warning dissemination system. Section 5 presents and discusses the obtained results. In Section 6 we review previous works closely related to our proposal, highlighting the main similarities and differences. Finally, Section 7 concludes this paper.

\section{Simulation Environment and Previous Concepts}

Simulation results presented in this paper were obtained using the ns-2 simulator (Fall and Varadhan, 2000), modified to consider the IEEE 802.11p standard $^{1}$. In terms of the physical layer, the data rate used for packet broadcasting is $6 \mathrm{Mbit} / \mathrm{s}$, as this is the maximum rate for broadcasting in $802.11 \mathrm{p}$ (Jiang et al., 2008). The MAC layer was also extended to include four different channel access priorities. Therefore, application messages are categorized into four different Access Categories (ACs), where AC0 has the lowest and AC3 the highest priority. The purpose of the $802.11 \mathrm{p}$ standard is to provide the minimum set of specifications required to ensure interoperability between wireless devices when attempting to communicate in potentially fast-changing communication environments. For our simulations, we chose the IEEE $802.11 \mathrm{p}$ because it is expected to be widely adopted by the industry.

The simulator was also modified to make use of our Real Attenuation and Visibility (RAV) scheme (Martinez et al., 2013), which proved to increase the level of realism in VANET simulations using real urban roadmaps in the presence of obstacles. The RAV propagation model is presented in detail in Section

\footnotetext{
${ }^{1}$ All these improvements and modifications are available at http://www.grc.upv.es/software/
} 
2.1. As for vehicular mobility, it has been obtained with CityMob for Roadmaps (C4R) (Fogue et al., 2012a), a mobility generator able to import maps directly from OpenStreetMap (OpenStreetMap, 2012), and make them available for being used by the ns-2 simulator.

To generate the movements for the simulated vehicles, we used the Krauss mobility model (Krauss et al., 1997) available in SUMO with some modifications to allow multi-lane behavior (Krajzewicz et al., 2012). This model is based on collision avoidance among vehicles by adjusting the speed of a vehicle to the speed of its predecessor using the following formula:

$$
v(t+1)=v_{1}(t)+\frac{g(t)-v_{1}(t) \tau}{\tau+1}+\eta(t),
$$

where $v$ represents the speed of the vehicle in $\mathrm{m} / \mathrm{s}, t$ represents the period of time in seconds, $v_{1}$ is the speed of the leading vehicle in $\mathrm{m} / \mathrm{s}, g$ is the gap to the leading vehicle in meters, $\tau$ is the driver's reaction time (set to 1 second in our simulations) and $\eta$ is a random numeric variable with a value between 0 and 1.

Our mobility simulations also account for areas with different vehicle densities. In a real town, traffic is not uniformly distributed; there are downtowns or points of interest that may attract vehicles. Hence, we include the ideas presented in the Downtown Model (Martinez et al., 2008) to add points of attraction in realistic roadmaps.

With regard to data traffic, vehicles operate in two modes: (a) warning mode, and (b) normal mode. Warning mode vehicles inform other vehicles about their status by sending warning messages periodically with the highest priority at the MAC layer; each vehicle is only allowed to propagate them once for each sequence number. Normal mode vehicles enable the diffusion of these warning messages and, periodically, they also send beacons with information such as their positions, speed, etc. These periodic messages have lower priority than warning messages, and so they are not propagated by other vehicles.

The roadmaps used in the simulations were selected in order to have differ- 
Table 1: Map features.

\begin{tabular}{|c|c|c|c|}
\hline Map & Streets & Junctions & SJ Ratio \\
\hline \hline Rome & 1655 & 1193 & 1.387 \\
\hline Valencia & 2829 & 2233 & 1.267 \\
\hline Sydney & 872 & 814 & 1.071 \\
\hline Amsterdam & 1494 & 1449 & 1.031 \\
\hline Los Angeles & 287 & 306 & 0.938 \\
\hline San Francisco & 725 & 818 & 0.886 \\
\hline Madrid & 628 & 715 & 0.878 \\
\hline
\end{tabular}

ent profile scenarios (i.e., with different topology characteristics). Table 1 and Figure 1 show the topology and the main features of the cities simulated, respectively. Note that we included a column labeled as SJ Ratio, which represents the result of dividing the number of streets between the number of junctions. As shown, the first four cities (Rome, Valencia, Sydney, and Amsterdam) present an SJ ratio greater than 1, which indicates that they have a complex topology, while the rest of the cities (Los Angeles, San Francisco, and Madrid) present a lower SJ value, which indicates that they have a simple topology.

We are interested in the following performance metrics: (i) percentage of informed vehicles, and (ii) number of messages received per vehicle. The percentage of informed vehicles is the percentage of vehicles that do receive the warning messages sent by warning mode vehicles. The number of messages received per vehicle (including beacons and warning messages) gives an estimation of channel contention and the overhead of the selected approach. Additionally, we are also interested in the warning notification time, which is the time required by normal vehicles to receive a warning message sent by a warning mode vehicle. This time allows us estimate the delivery delay.

In this work we performed more than 28,000 experiments, since we made 50 repetitions for each scenario while also varying the city roadmaps, the density of vehicles, and the broadcast scheme used. Due to space limitations, it is not possible to present the results of all of the cities simulated, so in some cases we only included the results obtained for San Francisco and Valencia since, 


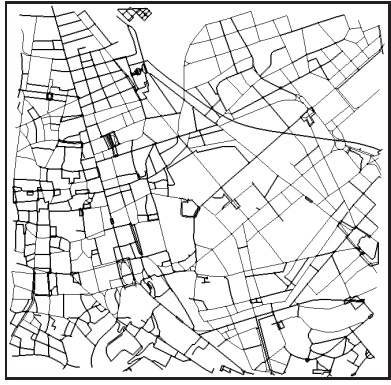

(a)

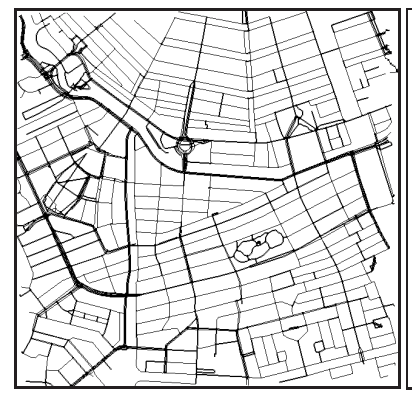

(d)

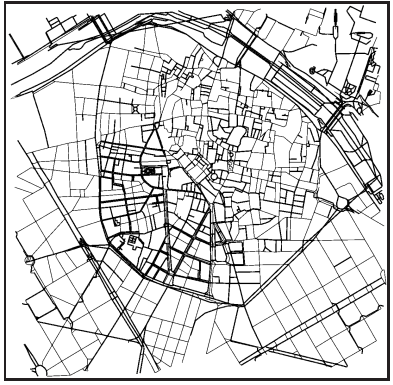

(b)

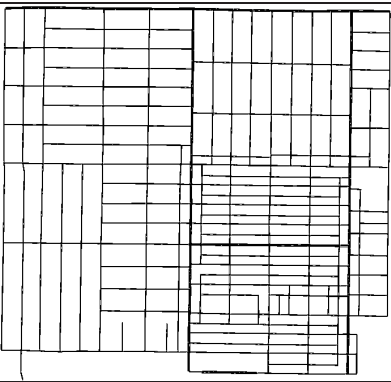

(e)

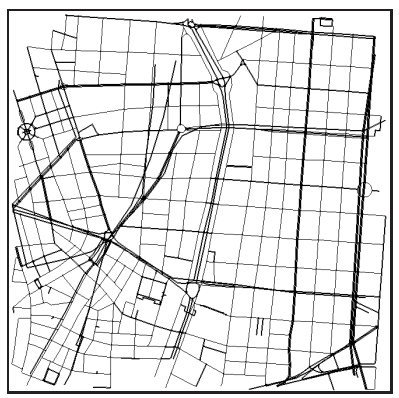

(g)

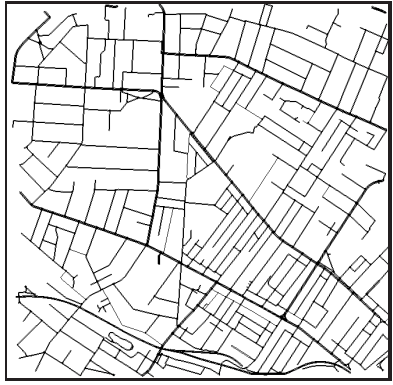

(c)

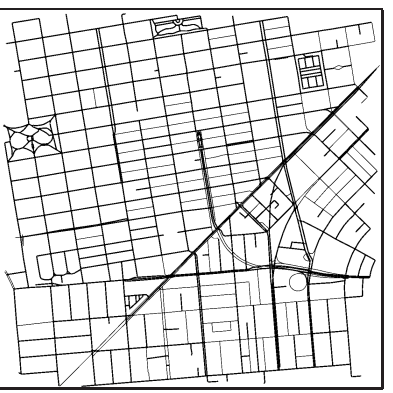

(f)

Figure 1: Scenarios selected in our simulations. Fragments of the cities of: (a) Rome (Italy), (b) Valencia (Spain), (c) Sydney (Australia), (d) Amsterdam (Netherlands), (e) Los Angeles (USA), (f) San Francisco (USA), and (g) Madrid (Spain). 
Table 2: Parameter settings in the simulations.

\begin{tabular}{|c|c|}
\hline Parameter & Value \\
\hline roadmaps & Rome, Valencia, Sydney, Amsterdam, \\
\hline number of vehicles per $\mathrm{km}^{2}$ & {$[25,50,100,150,200$, and 250$]$} \\
\hline roadmap size & $2000 m \times 2000 m$ \\
\hline warning message size & $256 B$ \\
\hline beacon message size & $512 B$ \\
\hline warning messages priority & $A C 3$ \\
\hline beacon priority & $A C 1$ \\
\hline interval between messages & 1 second \\
\hline $\mathrm{MAC} / \mathrm{PHY}$ & $802.11 \mathrm{p}$ \\
\hline radio propagation model & $R A V$ (Martinez et al., 2013) \\
\hline mobility model & Krauss (Krauss et al., 1997) \\
\hline channel bandwidth & $6 M b p s$ \\
\hline max. transmission range & $400 m$ \\
\hline$d_{\text {min }}$ (used in distance-based, eSBR, and eMDR) & $200 m$ \\
\hline simulation run & $120 \mathrm{~s}$ \\
\hline
\end{tabular}

according to our previous work (Sanguesa et al., 2013a), the simulation results obtained in these roadmaps are closer to the average ones. Table 2 shows the parameters used for the simulations.

In the next subsections we present some important concepts useful for our work. In particular, we first detail the Realistic Attenuation and Visibility (RAV), our proposed Radio Propagation Model (RPM) used in simulations; we also introduce the concept of neighbor in our system, which is a key factor to estimate the vehicle density at any instant by using vehicular networks; and finally, we show the vehicle density estimation function used in our approach.

\subsection{Real Attenuation and Visibility model for real roadmap scenarios}

A wireless signal propagation model can be characterized by: (a) attenuation schemes (signal power loss due to distance), and (b) visibility schemes (presence of obstacles interfering with signal propagation). The combination of these schemes makes up our radio propagation model, namely RAV.

Regarding attenuation, the RAV model implements signal attenuation due to the distance between vehicles as closely to reality as possible. In general, ns-2 
offers deterministic RPMs, i.e., the selected function determines the maximum distance a message could reach. If the receiver is within this range, the message will be successfully received; on the contrary, if the distance is greater, it will be lost. In order to increase realism, RAV uses a probabilistic approach to model packet losses due to channel noise and other situations. It uses a probability density function to determine the probability of a packet being successfully received at any given distance. With respect to other attenuation schemes, such as Two-Ray Ground and Nakagami, our scheme, instead of being theoretical, is obtained directly from experimental data (Martinez et al., 2013).

As for visibility, the main objective that a realistic visibility scheme should accomplish is to determine if there are obstacles between the sender and the receiver which interfere with the radio signal. In most cases, when using the $5.9 \mathrm{GHz}$ frequency band (used by the $802.11 \mathrm{p}$ standard), buildings absorb radio waves, and so communication is not possible. Hence, the RAV model allows vehicles communicating if one the following conditions are fulfilled:

- Sender and receiver are in line-of-sight, for example, if the two vehicles are located in the same street, or if they are located in different streets but there are no buildings blocking the radio signal.

- Sender and receiver are not in line-of-sight, but the receiver is close enough to a junction, increasing the probability of successful reception due to the reflection and diffraction of the wireless signal on the nearby buildings, as shown in Figure 2.

Figure 3 shows an example of our visibility scheme, where vehicle (A) is trying to disseminate a message. In that case, and assuming that any vehicle receiving a message will rebroadcast it the first time, the result will be vehicles B, C, D, and E receiving the message while the others (F, G, H, and I) will not receive it. The visibility scheme considers that radio signal can be propagated through streets and clear areas whereas the signal is mostly inhibited from passing through solid buildings. 


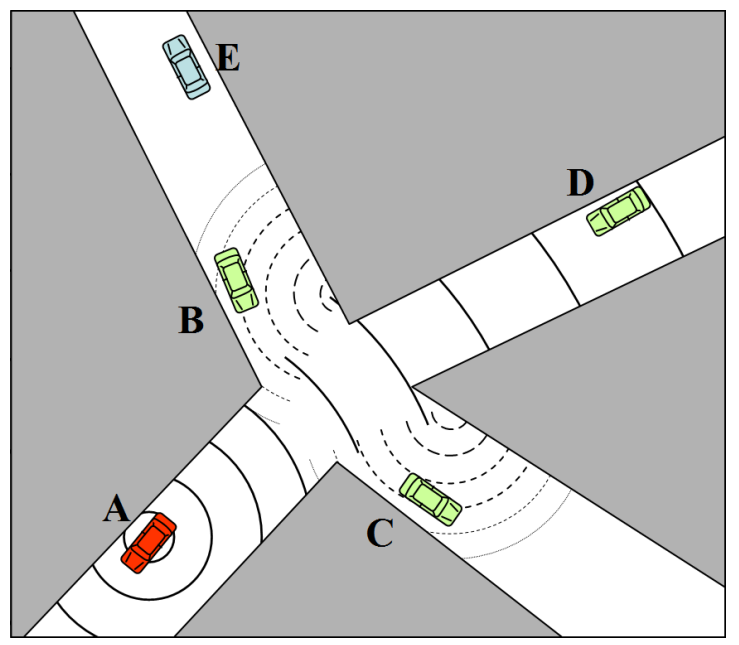

Figure 2: Vehicles in non-line-of-sight are able to receive the message through reflection and diffraction if they are close enough to a junction.

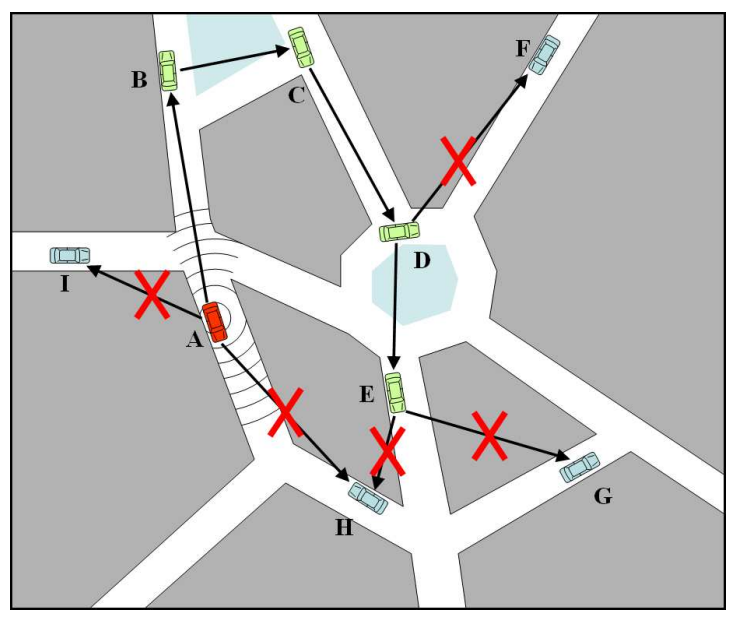

Figure 3: Our visibility scheme: example scenario. Dark polygons represent buildings and light areas are clear spaces between streets. 


\subsection{Neighbor concept}

In this work, we call neighbors those vehicles that are reachable by one-hop messages, without requiring any additional rebroadcast, i.e., they are within the communication range of the sender vehicle and the signal transmission is not blocked by any obstacle such as buildings. In our system, all the vehicles considered maintain a neighbor list that is built by using the beacons exchanged periodically among the vehicles, avoiding any additional channel overhead. Whenever a new beacon is received, each vehicle checks its neighbor list to determine if the sender is a new neighbor, thereby adding this vehicle to the list. The neighbors' list is also updated when new beacons are not received from a former neighbor after 2 seconds. In that case, the neighbor is removed from the list.

\subsection{Density Estimation Function}

Our proposed Real-time Adaptive Dissemination (RTAD) system relies on the Optimal Broadcast Selection Algorithm to adapt the dissemination process to the specific characteristics at a given time. However, the Optimal Broadcast Selection Algorithm needs to estimate the vehicle density to select the most appropriate broadcast scheme. In particular, vehicle density estimation is a determinant step when determining which is the best dissemination scheme to use.

In Sanguesa et al. (2013a), we proposed a method to calculate the density according to the number of beacons received by each vehicle. In this work, we have modified it to increase its accuracy. Specifically, the RTAD system uses the number of neighbors, instead of the number of beacons received, to accurately estimate the vehicle density.

To propose a method able to accurately estimate the density of vehicles, based on the number of beacons received and the roadmap topology, we made a total of 4,000 experiments. These experiments involved the simulation of controlled scenarios (i.e., scenarios where the actual density is known).

According to the results obtained, we propose a density estimation function capable of estimating the vehicular density in every urban environment, at any 
Table 3: Proposed equation coefficients.

\begin{tabular}{|c|c|}
\hline Coeff. & Value \\
\hline $\mathrm{a}$ & $-7.9174488563075374 \mathrm{E}+02$ \\
\hline $\mathrm{b}$ & $-6.5986335423139231 \mathrm{E}-01$ \\
\hline $\mathrm{c}$ & $2.2717144481059472 \mathrm{E}+03$ \\
\hline $\mathrm{d}$ & $1.1989021509173876 \mathrm{E}+00$ \\
\hline $\mathrm{f}$ & $-2.1019985100197723 \mathrm{E}+03$ \\
\hline $\mathrm{g}$ & $-1.7509026843351649 \mathrm{E}-02$ \\
\hline $\mathrm{h}$ & $6.3096785945693671 \mathrm{E}+02$ \\
\hline $\mathrm{i}$ & $-4.8107314165096247 \mathrm{E}+00$ \\
\hline $\mathrm{j}$ & $-7.6438506962308739 \mathrm{E}-01$ \\
\hline $\mathrm{k}$ & $1.4601116345732333 \mathrm{E}+01$ \\
\hline
\end{tabular}

instant of time. To this purpose, we performed a regression analysis (ZunZun, 2012) that allowed us to find the polynomial equation offering the best fit to the data obtained through simulation.

Equation 2 shows the density estimation function, which is able to estimate the number of vehicles per $\mathrm{km}^{2}$ in urban scenarios, according to the number of neighbors, and the SJ ratio (i.e., streets/junctions).

$$
f(x, y)=a+b x+c y+d x^{2}+f y^{2}+g x^{3}+h y^{3}+i x y+j x^{2} y+k x y^{2}
$$

In this equation, $x$ is the number of neighbors of each vehicle, and $y$ is the SJ ratio obtained from the roadmap. The values of the polynomial coefficients $(a, b, c, d, f, g, h, i, j$, and $k)$ are listed in Table 3.

Our new density estimation approach, based on the number of neighbors, yields more accurate results, presenting a lower sum for the squared absolute error (approximately $5.098 \mathrm{E}+03$, whereas the beacon-based approach resulted in 6.332E +03 (Sanguesa et al., 2013a)).

Figure 4 shows the density data obtained in the simulation for the roadmaps of San Francisco and Valencia, as well as the values estimated according the SJ Ratio (roadmap complexity) and the number of neighbors. As shown, we 


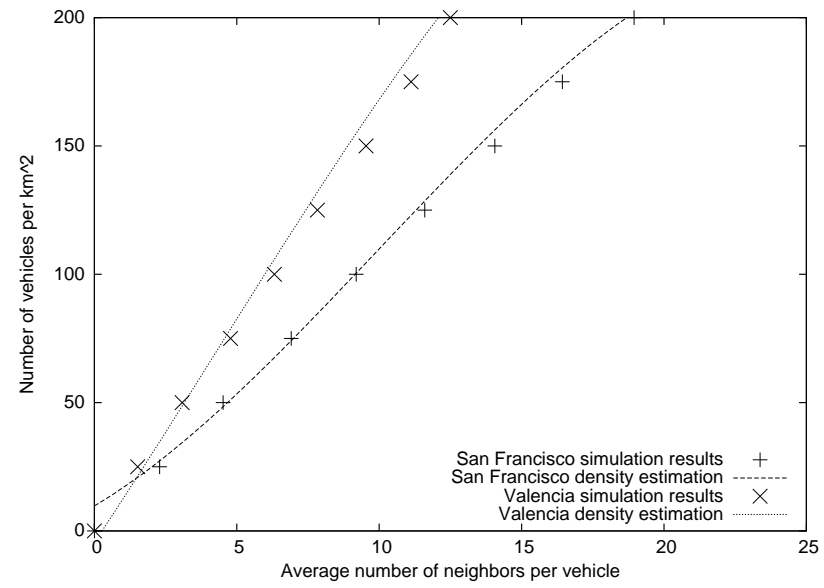

Figure 4: Simulation results and vehicle density estimation in San Francisco and Valencia.

achieve a good fit for the values obtained in the simulations, especially in San Francisco.

\section{RTAD: Analysis of the Optimal Broadcast Scheme}

One of the main characteristics of VANETs is the great variability of the conditions affecting each vehicle. Thus, broadcasting decisions taken should not remain immovable. Instead, the dissemination system should dynamically adapt its broadcasting policy to the specific characteristics and situations, thereby improving the whole dissemination process. In this work we propose RTAD, a real-time adaptive dissemination system specially designed for VANETs, in which each vehicle individually adopts a specific dissemination scheme according to each situation. In our proposed system, each vehicle is able to obtain and analyze the characteristics of the environment, thereby choosing the optimal diffusion policy in each situation. To select the optimal broadcast scheme for a specific scenario, RTAD accounts for two different performance metrics (i.e., the vehicle density and the roadmap topology), that allow it to determine which dissemination scheme to use at any time. 
In order to determine which are the optimal broadcast schemes that our RTAD can use for each particular scenario, in this section we first review the different broadcast schemes actually used by our adaptive system; then we present in detail the main metrics we use to measure the broadcast schemes' goodness, and finally, we introduce the optimal broadcast selection algorithm.

\subsection{Broadcast Schemes Used}

So far, several authors have proposed different dissemination schemes to mitigate broadcast storms (Tseng et al., 2002; Wisitpongphan et al., 2007; Suriyapaibonwattana and Pomavalai, 2008; Bi et al., 2010; Slavik and Mahgoub, 2010). However, all of these schemes consider free space environments where no blocking obstacles are considered at all. They have not addressed the impact of buildings and other urban obstacles on the wireless signal propagation in realistic urban scenarios. The consequences derived from those incomplete analyses can be observed when their performance is tested in realistic urban topologies, showing that they are unable to choose suitable relaying vehicles, or proving to be too restrictive to achieve an efficient dissemination (Martinez et al., 2010). Some of the most representative broadcast schemes are presented below.

- The Counter-based scheme (Tseng et al., 2002). Initially proposed for Mobile Ad Hoc Networks (MANETs), this scheme aims at mitigating broadcast storms by using a threshold $C$ and a counter $c$ to keep track of the number of times a broadcast message is received. Whenever $c \geq C$, rebroadcast is inhibited.

- The Distance-based scheme (Tseng et al., 2002). This scheme accounts for the relative distance $d$ between vehicles to decide whether to rebroadcast or not. When the distance $d$ between two vehicles is short, the additional coverage $(\mathrm{AC})$ area of the new rebroadcast is lower, and so rebroadcasting the warning message is not recommended. Forwarding is only beneficial when the additional coverage is nearly maximum. 
- The enhanced Street Broadcast Reduction (eSBR) (Martinez et al., 2010). This scheme is specially designed to be used in VANETs, taking advantage of the information provided by maps and built-in positioning systems, such as the GPS. Vehicles are only allowed to rebroadcast messages if they are located far from their source $\left(>d_{\min }\right)$, or if the vehicles are located in different streets, giving access to new areas of the scenario. The eSBR scheme uses information about the roadmap to avoid blind areas due the presence of urban structures blocking the radio signal.

- The enhanced Message Dissemination for Roadmaps (eMDR) (Fogue et al., 2012b). As an improvement to the eSBR scheme, eMDR increases the efficiency of the system by avoiding to forward the same message multiple times if nearby vehicles are located in different streets. Specifically, vehicles use the information about the junctions of the roadmap, and only the vehicle closest to the geographic center of the junction, according to the geopositioning system, is allowed to forward the messages received. This strategy aims at reducing the number of broadcasted messages while maintaining a high percentage of vehicles informed.

- The eMDR and eSBR schemes proved to be especially effective in sparse urban environments. However, the number of messages produced may become excessive in scenarios with a high vehicle density. To cope with this deficiency, in this paper we also propose a novel dissemination scheme called Nearest Junction Located (NJL) that is completely based on the topology of the roadmap, allowing vehicles to rebroadcast a message only if they are the nearest vehicle to the geographical coordinates of any junction obtained from the integrated maps. This scheme follows a procedure similar to the eMDR algorithm, although ignoring the distance between sender and receiver; thus, it only focuses on the location of the receiving vehicle. As shown in the two next subsections, although the performance of this algorithm is not optimal in sparse environments, it performs quite well in high-density scenarios where the dominant factor to improve the 
dissemination process is the position of the vehicles, achieving results similar to those obtained by the eMDR and eSBR schemes, while requiring only a fraction of the overall number of messages.

To better understand the operation of all these schemes (i.e., counter-based, distance-based, eSBR, eMDR, and NJL algorithms, we provide a formal definition of these schemes using set theory. In the mentioned formulation, the following notation is used:

- $\mathbb{V}$ : set of vehicles present in the scenario.

- $\mathbb{N}_{i}$ : set of neighbor vehicles of vehicle $v_{i} \in \mathbb{V}$.

- M: set of warning messages disseminated by vehicles.

- $\mathbb{J}$ : set of junctions of the road layout.

The definition of the algorithms requires some basic functions to express events and relationships between the components of the scenario. Specifically, the following functions are required:

- $\operatorname{recv}\left(v_{r}, v_{s}, m, t\right)$ : vehicle $v_{r}$ receives a warning message $m$ from vehicle $v_{s}$ at time $t$.

- $\operatorname{dist}\left(e_{1}, e_{2}\right)$ : Euclidean distance between elements $e_{1}$ and $e_{2}$, i.e.:

$$
\operatorname{dist}\left(e_{1}, e_{2}\right)=\sqrt{\left(e_{1} \cdot x-e_{2} \cdot x\right)^{2}+\left(e_{1} \cdot y-e_{2} \cdot y\right)^{2}}
$$

- rebroadcast $(v, m, t)$ : vehicle $v$ broadcasts a warning message $m$ at time $t$.

Equation 4 shows the formulation of the counter-based algorithm. In this scheme, after receiving a warning message from a vehicle $v_{s}$, the receiver $v_{r}$ would only rebroadcast the message if the message has been received less than a certain number of times, i.e., using a threshold $C$ and a counter $c$ to keep track of the number of times a broadcast message is received, whenever $c \geq C$, re-broadcast is inhibited. 


$$
\begin{aligned}
& \forall v_{r} \in \mathbb{V} \wedge \exists m \in \mathbb{M}, v_{s} \in \mathbb{V} \wedge \operatorname{recv}\left(v_{r}, v_{s}, m, t\right) \Rightarrow \\
& \left(\operatorname{rebroadcast}\left(v_{r}, m, t\right) \Leftrightarrow c<C\right)
\end{aligned}
$$

Equation 5 shows the formulation of the distance-based algorithm. In this scheme, after receiving a warning message from a vehicle $v_{s}$, the receiver $v_{r}$ would only rebroadcast the message if the distance between the sender vehicle $v_{s}$ and the receiver vehicle $v_{r}$ is greater than a minimum rebroadcast distance $d_{\text {min }}$.

$$
\begin{aligned}
& \forall v_{r} \in \mathbb{V} \wedge \exists m \in \mathbb{M}, v_{s} \in \mathbb{V} \wedge \operatorname{recv}\left(v_{r}, v_{s}, m, t\right) \Rightarrow \\
& \left(\operatorname{rebroadcast}\left(v_{r}, m, t\right) \Leftrightarrow \operatorname{dist}\left(v_{r}, v_{s}\right)>d_{\text {min }}\right)
\end{aligned}
$$

Equation 6 shows the formulation of the eSBR algorithm. As can be observed, after receiving a warning message from a vehicle $v_{s}$, each vehicle $v_{r}$ rebroadcasts the message if the distance between the sender vehicle $v_{s}$ and the receiver vehicle $v_{r}$ is greater than a minimum rebroadcast distance $d_{m i n}$, set in our simulations to $200 \mathrm{~m}$; or if $v_{r}$ is located near a junction $j$, giving access to new streets possibly blocked by the effect of buildings on radio signal. A threshold $t h_{j}$ of $20 \mathrm{~m}$ is used to determine whether the vehicle is located near a junction in the map.

$$
\begin{aligned}
& \forall v_{r} \in \mathbb{V} \wedge \exists m \in \mathbb{M}, v_{s} \in \mathbb{V} \wedge \operatorname{recv}\left(v_{r}, v_{s}, m, t\right) \Rightarrow \\
& \left(\operatorname{rebroadcast}\left(v_{r}, m, t\right) \Leftrightarrow \operatorname{dist}\left(v_{r}, v_{s}\right)>d_{\text {min }} \vee\left(\exists j \in \mathbb{J} \wedge \operatorname{dist}\left(v_{r}, j\right)<t h_{j}\right)\right)
\end{aligned}
$$

Equation 7 contains the operation of the eMDR algorithm. The main difference between eSBR and eMDR lies on the number of vehicles allowed to retransmit in junctions. Whereas all the vehicles located in junctions are allowed rebroadcasting in eSBR, eMDR only allows one vehicle per junction as a forwarding node. To achieve this behavior, each vehicle $v_{r}$ stores a list $\mathbb{N}_{r}$ 


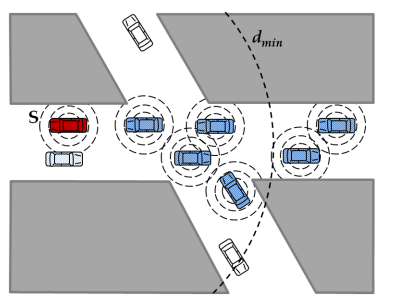

(a)

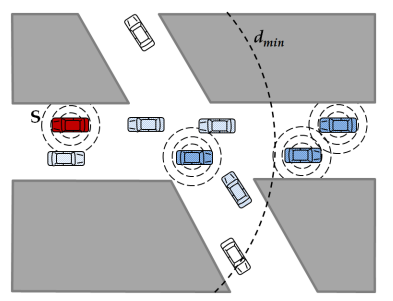

(b)

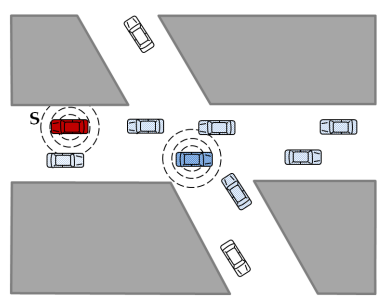

(c)

Figure 5: Comparison of different dissemination schemes for VANETs: (a) eSBR, (b) eMDR, and (c) NJL.

containing its neighbors in communication range, built by means of periodic beacons sent by all vehicles. Hence, vehicle $v_{r}$ only rebroadcasts the message if the distance to sender is greater than $d_{\min }$, or if it is located near a junction and it is the closest vehicle to the center of the junction, obtained from its geographical coordinates of GPS integrated maps.

$$
\begin{aligned}
& \forall v_{r} \in \mathbb{V} \wedge \exists m \in \mathbb{M}, v_{s} \in \mathbb{V} \wedge \operatorname{recv}\left(v_{r}, v_{s}, m, t\right) \Rightarrow \\
& \left(\operatorname{rebroadcast}\left(v_{r}, m, t\right) \Leftrightarrow \operatorname{dist}\left(v_{r}, v_{s}\right)>d_{\text {min }} \vee\right. \\
& \left.\left(\exists j \in \mathbb{J} \wedge \operatorname{dist}\left(v_{r}, j\right)<t h_{j} \wedge\left(\nexists v_{n} \in \mathbb{N}_{r} \wedge \operatorname{dist}\left(v_{n}, j\right)<\operatorname{dist}\left(v_{r}, j\right)\right)\right)\right)
\end{aligned}
$$

Finally, Equation 8 shows the formulation of the NJL algorithm. As shown, NJL ignores the distance between sender and receiver and only allows rebroadcasting if the receiver vehicle is the closest to the geographical center of the junction with respect to its neighbors.

$$
\begin{aligned}
& \forall v_{r} \in \mathbb{V} \wedge \exists m \in \mathbb{M}, v_{s} \in \mathbb{V} \wedge \operatorname{recv}\left(v_{r}, v_{s}, m, t\right) \Rightarrow \\
& \left(\operatorname { r e b r o a d c a s t } ( v _ { r } , m , t ) \Leftrightarrow \left(\exists j \in \mathbb{J} \wedge \operatorname{dist}\left(v_{r}, j\right)<t h_{j} \wedge\right.\right. \\
& \left.\left.\left(\nexists v_{n} \in \mathbb{N}_{r} \wedge \operatorname{dist}\left(v_{n}, j\right)<\operatorname{dist}\left(v_{r}, j\right)\right)\right)\right)
\end{aligned}
$$

Figure 5 shows graphically the differences between eSBR, eMDR, and NJL schemes in a specific VANET scenario, where vehicle $S$ broadcasts a warning 
message. The line labeled as $d_{\min }$ represents the minimum rebroadcast distance used by eSBR and eMDR. Darker vehicles will be allowed to forward the messages received from $S$, and it is noticeable how eSBR is the less restrictive scheme, whereas NJL is the most restrictive one, and thus more suitable for scenarios with a high vehicle density.

\subsection{Metric 1: Percentage of Informed Vehicles}

During the warning message dissemination process, the most important objective to accomplish consists on informing the highest number of vehicles in the shortest time possible. In particular, to better assess the optimal dissemination scheme to be used at any time, we performed several experiments using roadmaps with different features, as well as varying the density of vehicles. Figure 6 presents the evolution of the dissemination process in terms of informed vehicles for the maps of San Francisco and Valencia under four different vehicle densities: 25, 100, 150, and 250 vehicles $/ \mathrm{km}^{2}$.

It is noticeable how the roadmap topology and the vehicle density are determinant factors affecting the performance of the dissemination process. In general, the dissemination process develops faster (i.e., more vehicles are informed during a same period) when the vehicle density increases, independently from the broadcast scheme used, and especially in complex roadmaps. For sparse urban scenarios, the counter-based scheme provides the best results in terms of informed vehicles, whereas for densities above 150 vehicles $/ \mathrm{km}^{2}$, the dissemination process presents a very similar behavior for all the selected broadcast schemes. The exception is the distance-based scheme in the map of Valencia, which proved to be very inefficient due to the high amount of obstacles interfering with the radio signal, as this roadmap presents a higher complexity (the SJ Ratio is higher than San Francisco).

In addition, we corroborated that simple and regular city profiles, like San Francisco, allow an easier propagation of the radio signal, increasing the number of informed vehicles at a given time. However, the most restrictive schemes, such as NJL, require a very high density of vehicles to achieve an efficiency similar 


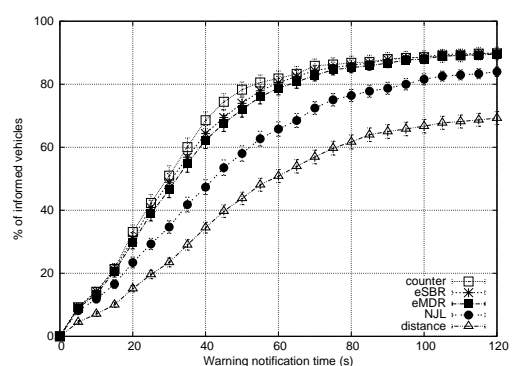

(a)

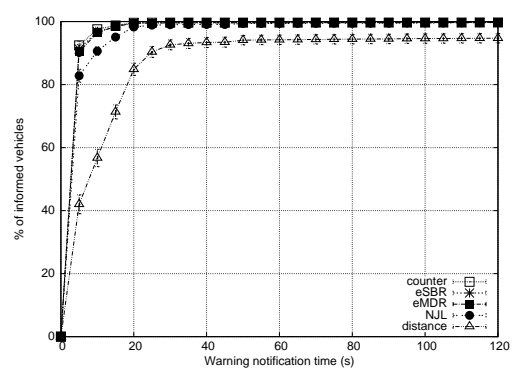

(b)

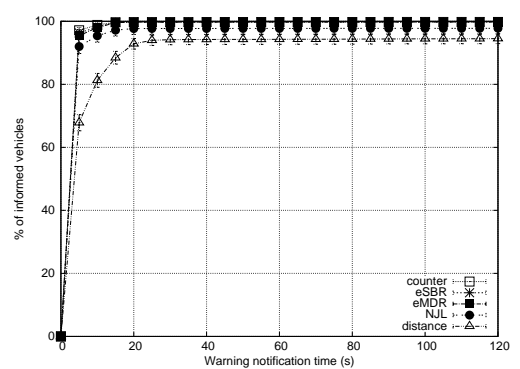

(c)

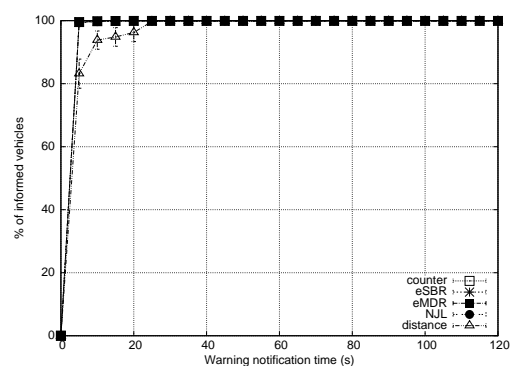

(d)

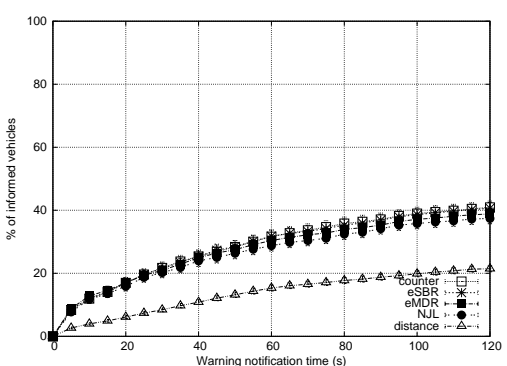

(e)

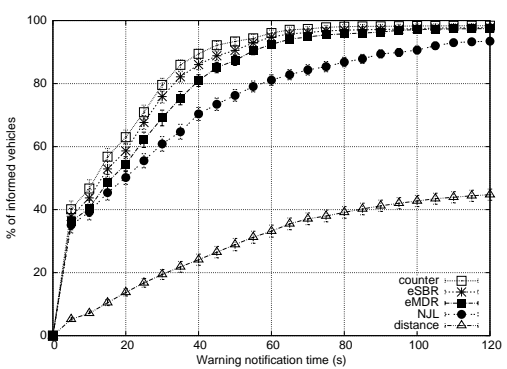

(f)

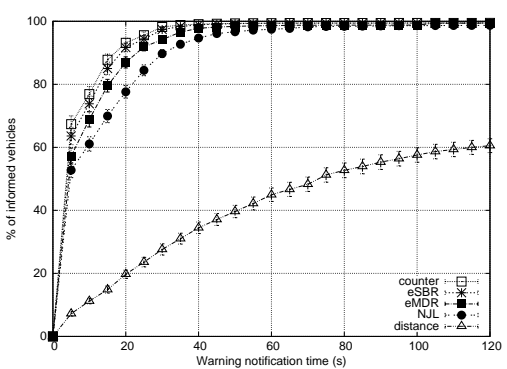

(g)

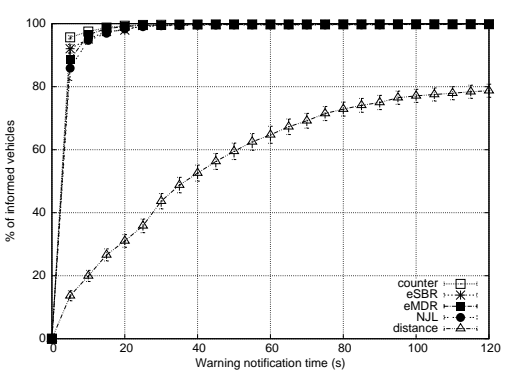

(h)

Figure 6: Percentage of informed vehicles in San Francisco for: (a) 25, (b) 100, (c) 150, and (d) 250 vehicles $/ \mathrm{km}^{2}$, as well as in Valencia for: (e) 25, (f) 100, (g) 150, and (h) 250 vehicles $/ \mathrm{km}^{2}$. 
to other dissemination schemes.

These results indicate that basing the broadcast policy selection only on the percentage of informed vehicles (e.g., after some minutes) could lead to wrong decisions that could seriously affect the efficiency of the system, justifying the need for additional metrics to perform the broadcast scheme selection. Additionally, to better characterize the dissemination process over time, we consider that the broadcast selection should account for the percentage of informed vehicles at different time instants $\left(\operatorname{In} f_{T}\right)$. Specifically, we propose to measure the percentage of vehicles receiving warning messages after 10, 30, and 120 seconds (i.e., $\operatorname{In} f_{10}, \operatorname{In} f_{30}$, and $\left.\operatorname{In} f_{120}\right)$ since it is important to account for the first seconds from the time when the dangerous situation started being notified until the dissemination stabilizes. This provides information about both the speed and completeness of the dissemination process. The first 10 seconds provide a good reference of the dissemination speed, the second period (30 seconds) offers a balance between dissemination speed and the completeness, and the state of the scenario after 120 seconds shows the stationary value when no evolution is observed.

These three values are combined according to Equation (9), to provide a single value representing the efficiency of the dissemination process $\left(P_{\text {inf }}\right)$ by each broadcast scheme $(b)$.

$$
P_{i n f}(b)=\frac{\sum_{t \in T} \operatorname{In} f_{t}(b)}{|T|}
$$

\subsection{Metric 2: Messages Received per Vehicle}

The number of messages produced by a given dissemination scheme may become very important in VANETs due to the high number of messages sent and received by the vehicles involved. This could increase channel contention and the frequency of collisions. Therefore, a reduction of the number of messages received per vehicle under this situation, without reducing the percentage of informed vehicles, will improve the warning message dissemination process, 
allowing other applications sharing the channel to operate adequately. To this end, it is necessary to evaluate the different dissemination schemes, taking into account the number of messages received by each vehicle in order to select the optimal scheme for each particular scenario.

Figure 7 shows the number of messages received per vehicle in San Francisco and Valencia. Notice that the selected dissemination scheme presents a determinant influence over the amount of messages received; some of them show only a fraction of the messages required by other schemes. In general, the counterbased scheme produces the highest number of messages, whereas the distancebased scheme is the most restrictive one. As we might suppose, the NJL scheme produces the smallest amount of messages of all the schemes which used the information topology of the map to select the forwarding nodes. Note that NJL generates fewer messages than the distance-based algorithm in San Francisco's high density scenarios. Again, the features of the map are determinant for the performance of the system. Simple maps allow a faster dissemination at the cost of noticeably increasing the number of messages received per vehicle, thereby increasing the probability of broadcast storms. Thus, more restrictive schemes are recommended for this kind of roadmaps.

We consider that the number of messages received per vehicle $\left(M_{\text {recv }}\right)$ is an important metric to be accounted for when ensuring an efficient dissemination process. If the wireless channel is saturated with packets, the high degree of contention and the occurrence of collisions will reduce the performance of the process, producing broadcast storms. Hence, the number of messages must remain as low as possible without compromising the dissemination efficiency.

\subsection{Optimal Broadcast Selection Algorithm}

The core of our RTAD system is the Optimal Broadcast Selection Algorithm which makes use of the two metrics presented before $\left(P_{\text {inf }}\right.$ and $\left.M_{\text {recv }}\right)$ to select the broadcast scheme to be used on each particular situation. Specifically, it works following a three step process, as shown in Algorithm 1: 


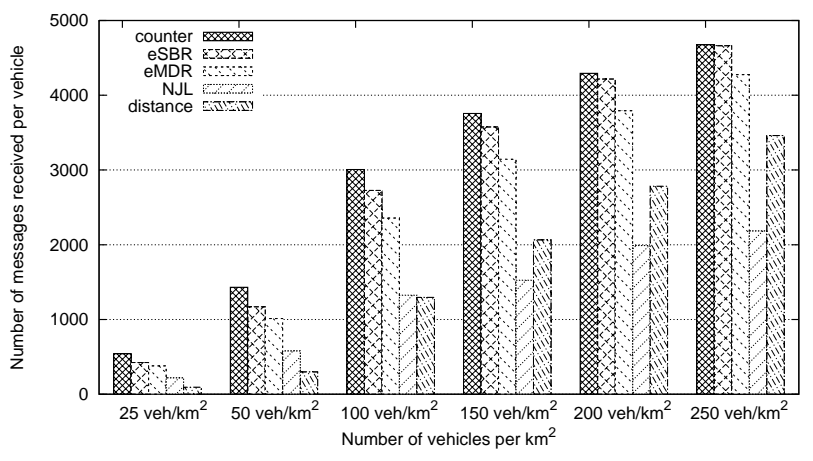

(a)

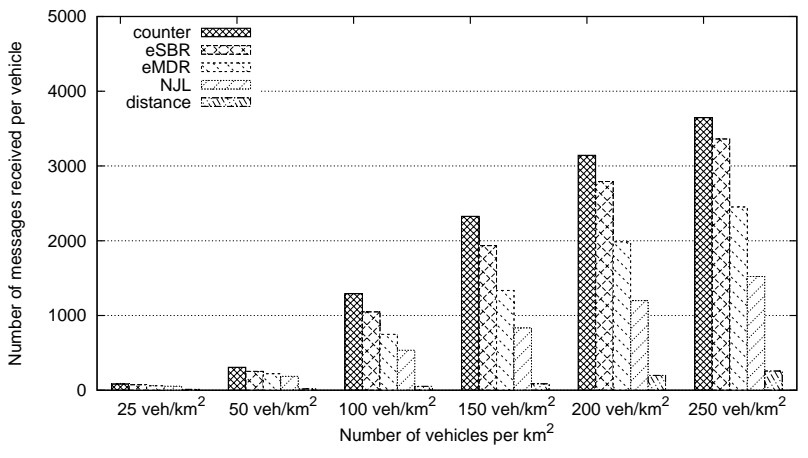

(b)

Figure 7: Number of messages received per vehicle when varying the broadcast scheme and the vehicular density in: (a) San Francisco and (b) Valencia. 


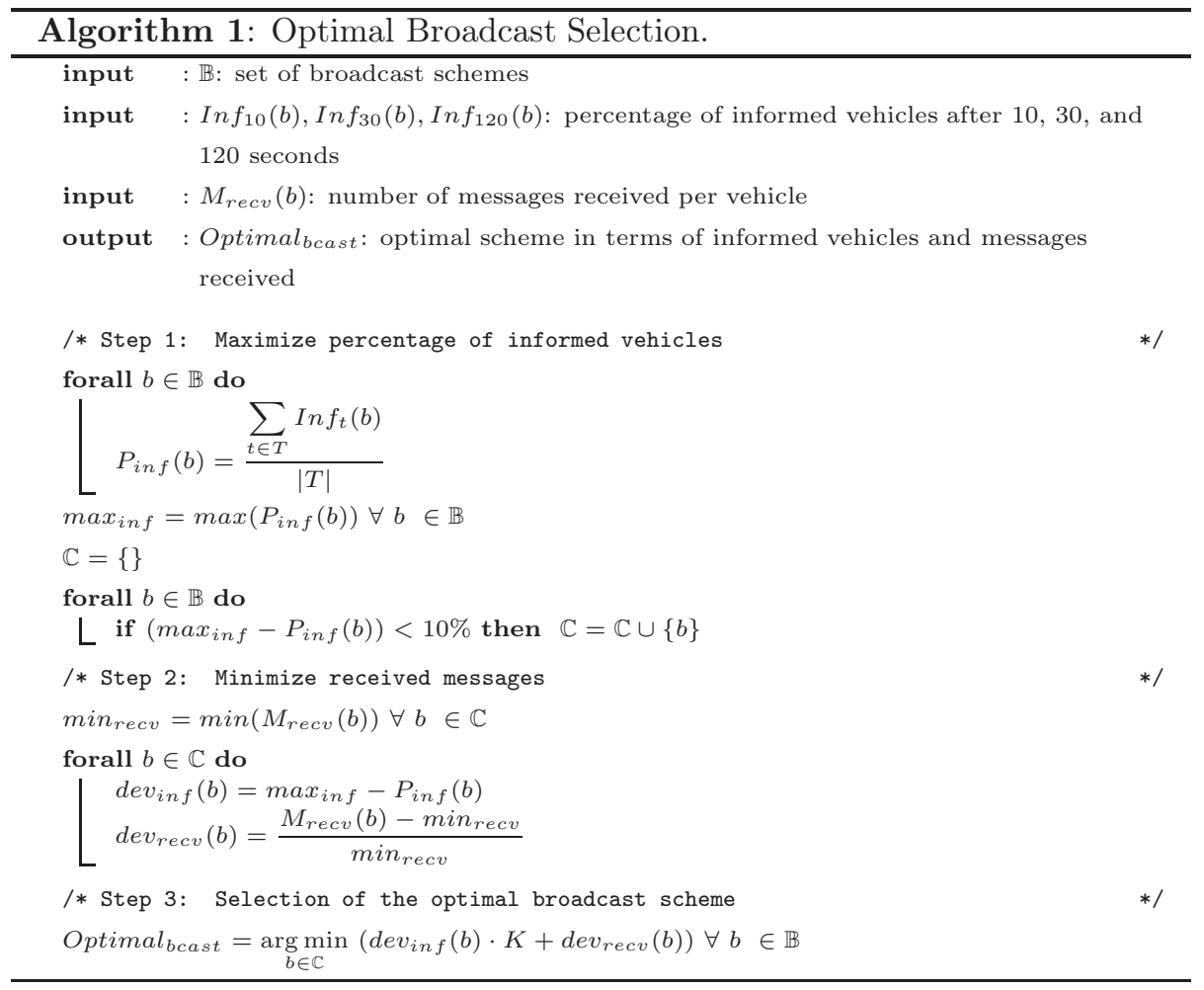


- Step 1: For each considered broadcast scheme, the first metric $\left(P_{\text {inf }}\right)$ is computed, and the scheme with the highest percentage of informed vehicles in the shortest time is selected. Due to the importance of this metric, only the dissemination schemes with a deviation lower than $10 \%$ with respect to the best one are considered for the second step of the algorithm, and then included in set $\mathbb{C}$.

- Step 2: Considering only the broadcast schemes in $\mathbb{C}$, the scheme producing the lowest number of messages per vehicle $\left(M_{\text {recv }}\right)$ is obtained, in order to reduce the probability of broadcast storms, and the percentage variation with respect to this value is computed for each scheme.

- Step 3: The optimal scheme will be selected as the one minimizing the deviation with respect to both the maximal $P_{\text {inf }}$ and the minimal $M_{\text {recv }}$. Depending on the vehicle density, our proposed algorithm adapts its behavior. Hence, it is more important to reduce the number of messages received in high vehicle densities, whereas increasing the number of informed vehicles becomes more important in sparse scenarios, where the number of messages received is not a problem. Specifically, our algorithm varies the degree of importance of the two metrics (i.e., $P_{\text {inf }}$ and $M_{\text {recv }}$ ) by using the $K$ value, calculated as follows:

$$
K=\frac{100}{\text { density of vehicles(vehicles } \left./ \mathrm{km}^{2}\right)}
$$

In particular, we used the value of reference 100 vehicles $/ \mathrm{km}^{2}$ to compute $K$, since our experiments showed that the differences in terms of informed vehicles decrease noticeably for densities above 100 vehicles $/ \mathrm{km}^{2}$ (see Figure 6), and, hence, a higher weight is assigned to the number of messages received when this density is exceeded. To sump up, we use $K$ in order to promote each metric depending on the current density, promoting the percentage of informed vehicles when densities are low, and trying to reduce the number of messages when densities are higher, and the differences in terms of informed vehicles are minimal. 
Table 4: Simulation results for 100 vehicles $/ \mathrm{km}^{2}$ in Valencia.

\begin{tabular}{|c|c|c|c|c|c|c|c|c|c|c|}
\hline Broadcast & $\operatorname{In} f_{10}$ & $\operatorname{In} f_{30}$ & Inf $f_{120}$ & $P_{\text {inf }}$ & $d e v_{i n f}$ & $\begin{array}{c}\mathbb{C}_{1} \\
\text { (Step 1) }\end{array}$ & $M_{\text {recv }}$ & $\begin{array}{c}d e v_{r e c v} \\
\text { (Step 2) }\end{array}$ & $d e v_{T o t}$ & $\begin{array}{l}\text { Optimal } \\
\text { (Step 3) }\end{array}$ \\
\hline Counter & $46.6 \%$ & $79.5 \%$ & $98.3 \%$ & 74.80 & $0 \%$ & $v$ & 1196 & $77.93 \%$ & 77.93 & $x$ \\
\hline Distance & $7.10 \%$ & $19.4 \%$ & $44.7 \%$ & 23.73 & $68.27 \%$ & * & - & - & - & - \\
\hline eSBR & $43.7 \%$ & $75.8 \%$ & $97.7 \%$ & 72.40 & $3.21 \%$ & $\checkmark$ & 940 & $39.87 \%$ & 43.08 & $x$ \\
\hline eMDR & $40.4 \%$ & $69 \%$ & $68.93 \%$ & 60.38 & $7.84 \%$ & $\checkmark$ & 672 & $0 \%$ & 14.00 & $v$ \\
\hline NJL & $39.2 \%$ & $60.8 \%$ & $93.4 \%$ & 64.47 & $13.81 \%$ & * & - & - & - & - \\
\hline
\end{tabular}

Table 5: Broadcast Scheme Selected According to our Optimal Broadcast Selection Algorithm.

\begin{tabular}{|c|c|c|c|c|c|c|c|}
\hline \multirow{2}{*}{ City } & \multirow{2}{*}{ SJ Ratio } & \multicolumn{6}{|c|}{ Vehicle Density $\left(\mathbf{v e h} \cdot \mathbf{k m}^{2}\right)$} \\
\cline { 3 - 8 } & & 25 & 50 & 100 & 150 & 200 & 250 \\
\hline \hline Rome & 1.387 & eSBR & eSBR & eSBR & eMDR & NJL & NJL \\
\hline Valencia & 1.267 & eMDR & eMDR & eMDR & NJL & NJL & NJL \\
\hline Sydney & 1.071 & eMDR & eMDR & eMDR & NJL & NJL & NJL \\
\hline Amsterdam & 1.031 & eMDR & eMDR & NJL & NJL & NJL & NJL \\
\hline Los Angeles & 0.938 & eMDR & eMDR & NJL & NJL & NJL & NJL \\
\hline San Francisco & 0.886 & eMDR & eMDR & NJL & NJL & NJL & NJL \\
\hline Madrid & 0.878 & eMDR & eMDR & NJL & NJL & NJL & NJL \\
\hline
\end{tabular}

Table 4 contains an example of the performance of our broadcast scheme selection algorithm. Specifically, it shows the results obtained for Valencia when simulating 100 vehicles $/ \mathrm{km}^{2}$. All the values are obtained as the average of 50 repetitions for each configuration. It is noticeable how only three of the selected schemes are considered after the first step of the algorithm (i.e., the counterbased, the eSBR, and the eMDR broadcast schemes). Since the eMDR produces the lowest pondered cumulative deviation $\left(d e v_{T o t}\right)$, our algorithm considers it as the best broadcast scheme for this particular scenario.

After performing an offline analysis including all the studied scenarios and vehicle densities, Table 5 shows the selected broadcast scheme for each of the simulated scenarios according to our proposed Optimal Broadcast Selection Algorithm. Notice that the proposed NJL scheme is selected as the optimal one in most cases, especially under high vehicle densities or simple maps with a small SJ ratio, where the radio signal can cover large distances and broadcast storms 


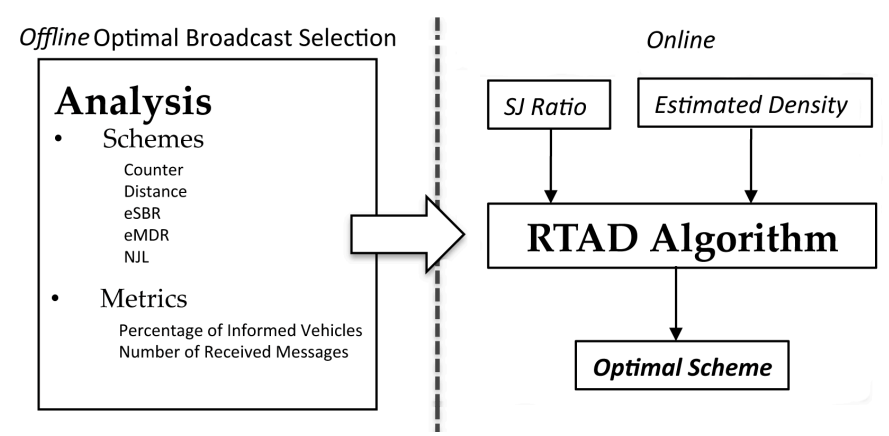

Figure 8: Details of our Real-time adaptive dissemination system.

are prone to occur. On the contrary, eMDR and eSBR schemes offer better results in scenarios where broadcast storms are not a problem, and the main objective is informing as many vehicles as soon as possible.

It is remarkable that almost all the schemes selected by our proposed algorithm rely on topology information to select the most appropriate forwarding vehicle, highlighting the importance of this factor in the warning dissemination process. In fact, broadcast schemes that only make use of the distance between the sender and the receiver, or which only focus on avoiding repeated messages, present a worse trade-off between performance and the amount of messages required.

\section{RTAD: Real-time Adaptive Dissemination System for VANETs}

As previously commented, the main objective of this work is to propose a real-time adaptive dissemination system in which each vehicle dynamically adopts a specific dissemination scheme according to each particular scenario. Based on the conclusions drawn in Section 3, now we present RTAD, our adaptive approach to improve message dissemination in VANETs.

Figure 8 shows how our proposal has been developed. First, we analyzed the different broadcast dissemination schemes in order to determine the optimal scheme to each specific situation. According to this analysis, we proposed a 


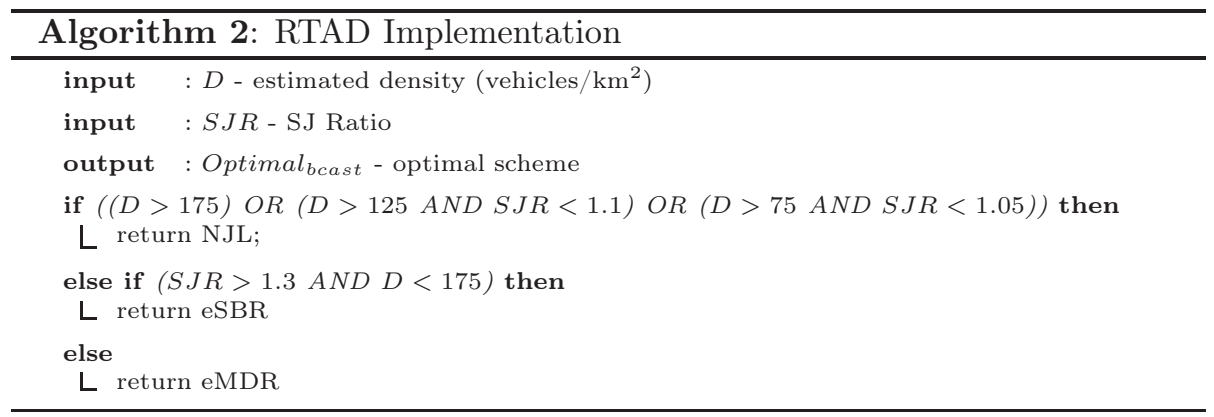

real-time adaptive system that makes each vehicle to automatically adopt the optimal dissemination scheme in order to fit the warning message delivery policy to each specific situation.

Algorithm 2 details the RTAD operation. As shown, RTAD determines which dissemination approach to use depending on the SJ Ratio and the vehicle density estimated, accordingly to the results previously obtained in the offline analysis that we performed. The SJ Ratio is automatically calculated by each vehicles by means of its geographical coordinates of GPS and the integrated maps, whereas the vehicle density is estimated in real time. According to our RTAD algorithm, each vehicle would adopt the optimal dissemination scheme in order to improve the dissemination process. The computational cost of this algorithm is very low, hence the overhead is almost negligible.

\section{RTAD Performance Evaluation}

To assess the performance of our proposal, we have performed experiments using several cities with different characteristics. In this section we present the results obtained in four cities: in one hand, we used Amsterdam and Los Angeles which were previously used to calibrate the proposal. On other hand, we also tested Sydney but with the aim of increasing the level of realism, we also considered that vehicles are not uniformly distributed since, in a real town, traffic is not uniformly distributed; instead, there are downtowns or points of interest that may attract vehicles. Specifically, we considered a downtown of 
750x750 meters located at the center of the map, which presents a higher density of vehicles. Finally, to better assess our proposal, we have also simulated a bigger area (i.e., $9 \mathrm{~km}^{2}$ ) of Santiago de Chile. This roadmap presents a SJ Ratio of 0.972 , so it can be considered a medium complexity map. Additionally, in these experiments we considered two different downtowns, the first one in the top right corner, and the second one in the bottom left corner.

Figure 9 shows the dissemination scheme selected by each vehicle at two different instants of the simulation ( 1 and 60 seconds after the simulation start), when simulating 150 vehicles $/ \mathrm{km}^{2}$ in Sydney. As shown, only 1 second after the simulation starts, the vehicles immediately proceed to adapt their broadcast mode (in particular, they use eMDR and NJL), according to the roadmap topology and the number of neighbors detected at this moment (as previously presented in Table 5). Note that this situation evolves, and after 60 seconds (see Figure 9.b), most of the vehicles are using the NJL scheme (a more restrictive dissemination scheme) since the warning messages can easily reach more vehicles in high density areas; however, isolated vehicles still use eMDR since they try to inform more vehicles without provoking broadcast storms.

Figure 10 shows the broadcast used by each vehicle 30 seconds after the beginning of the simulation. As expected, most of the vehicles located within a downtown use the NJL scheme, whereas the vehicles in the outskirts use a less restrictive dissemination policy; in particular, they use the eMDR scheme. Since the vehicle density is not uniformly distributed in the scenario, by using RTAD, each vehicle adapts its dissemination policy to better inform the rest of vehicles while significantly reducing the number of messages sent, thus avoiding overloading the channel.

Next, in order to assess RTAD goodness, we compare our approach with respect to two static dissemination schemes (eMDR and NJL), and three adaptive dissemination systems (UV-CAST, DV-CAST, and FDPD), previously proposed in the literature. 


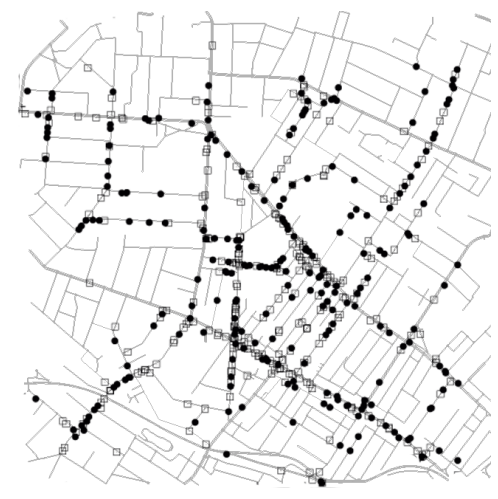

(a)

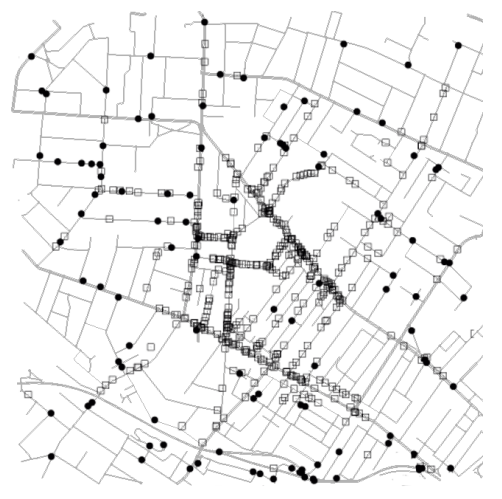

(b)

Figure 9: Broadcast scheme used by each vehicle in Sydney when simulating $150 \mathrm{veh} . / \mathrm{km}^{2}$ at: (a) $1 \mathrm{~s}$, and (b) $60 \mathrm{~s}$. after simulation start, respectively. The eMDR is represented with filled dots, and the NJL with empty squares.

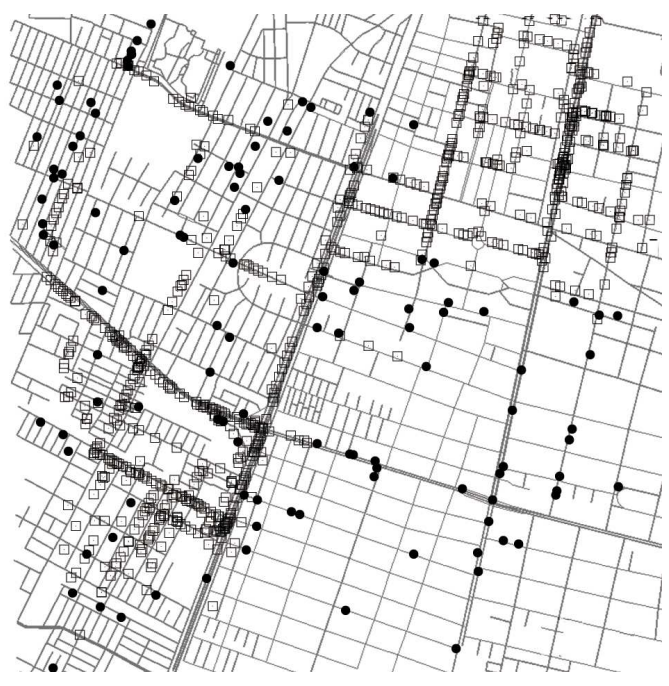

Figure 10: Broadcast scheme used by each vehicle in Santiago de Chile when simulating 100 veh. $/ \mathrm{km}^{2}, 30 \mathrm{~s}$. after the simulation start. The eMDR scheme is represented with filled dots, and the NJL scheme with empty squares. 
Table 6: Performance of the different dissemination schemes when varying the vehicle density and the city roadmap.

\begin{tabular}{|c|c|c|c|c|c|c|c|}
\hline \multirow[b]{2}{*}{$\mathrm{v} / \mathrm{km}^{2}$} & \multirow[b]{2}{*}{ Bcast } & \multicolumn{2}{|c|}{ Los Angeles } & \multicolumn{2}{|c|}{ Amsterdam } & \multicolumn{2}{|c|}{ Sydney } \\
\hline & & $P_{I n f}$ & $M_{r e c v}$ & $P_{I n f}$ & $M_{r e c v}$ & $P_{I n f}$ & $M_{\text {recv }}$ \\
\hline & RTAD & 54.30 & 261.43 & 37.33 & 154.08 & 33.47 & 112.55 \\
\hline \multirow[t]{2}{*}{25} & NJL & $45.07(-9.23 \%)$ & $163.81(-37.3 \%)$ & $32.47(-4.87 \%)$ & $89.55(-41.9 \%)$ & $29.43(-4.03 \%)$ & $71.24(-36.7 \%)$ \\
\hline & eMDR & $53.90(-0.40 \%)$ & $268.00(+2.5 \%)$ & $37.57(+0.23 \%)$ & $159.19(+3.3 \%)$ & $33.40(-0.07 \%)$ & $113.21(+0.6 \%)$ \\
\hline & RTAD & 86.33 & 789.70 & 61.00 & 470.20 & 55.07 & 360.68 \\
\hline \multirow[t]{2}{*}{50} & NJL & $75.00(-11.33 \%)$ & $556.89(-29.5 \%)$ & $54.83(-6.17 \%)$ & $290.26(-38.3 \%)$ & $48.87(-6.20 \%)$ & $231.58(-35.8 \%)$ \\
\hline & eMDR & $86.13(-0.20 \%)$ & $1017.42(+28.8 \%)$ & $63.23(+2.23 \%)$ & $666.49(+41.7 \%)$ & $56.17(+1.10 \%)$ & $458.67(+27.2 \%)$ \\
\hline \multirow{3}{*}{100} & RTAD & 97.63 & 1294.83 & 92.53 & 1051.98 & 82.23 & 816.05 \\
\hline & NJL & $95.47(-2.17 \%)$ & $1113.21(-14.0 \%)$ & $89.53(-3.00 \%)$ & $877.53(-16.6 \%)$ & $76.93(-5.30 \%)$ & $631.03(-22.7 \%)$ \\
\hline & eMDR & $98.43(+0.80 \%)$ & $2220.96(+71.5 \%)$ & $92.43(-0.10 \%)$ & $1786.42(+69.8 \%)$ & $84.27(+2.03 \%)$ & $1344.19(+64.7 \%)$ \\
\hline \multirow{3}{*}{150} & RTAD & 99.63 & 1605.60 & 97.53 & 1411.88 & 90.40 & 1092.46 \\
\hline & NJL & $99.50(-0.13 \%)$ & $1457.89(-9.2 \%)$ & $90.97(-6.57 \%)$ & $1173.85(-16.9 \%)$ & $89.33(-1.07 \%)$ & $918.73(-15.9 \%)$ \\
\hline & eMDR & $99.63(+0.00 \%)$ & $3096.48(+92.9 \%)$ & $97.60(+0.07 \%)$ & $2538.63(+79.8 \%)$ & $92.47(+2.07 \%)$ & $2095.19(+91.8 \%)$ \\
\hline & RTAD & 99.73 & 1773.84 & 98.57 & 1666.42 & 96.77 & 1331.97 \\
\hline \multirow[t]{2}{*}{200} & NJL & $99.63(-0.10 \%)$ & $1602.39(-9.7 \%)$ & $98.97(+0.40 \%)$ & $1599.83(-4.0 \%)$ & $93.20(-3.57 \%)$ & $1136.45(-14.7 \%)$ \\
\hline & eMDR & $99.73(+0.00 \%)$ & $3738.32(+110.7 \%)$ & $99.17(+0.60 \%)$ & $3284.05(+97.1 \%)$ & $94.57(-2.20 \%)$ & $2661.47(+99.8 \%)$ \\
\hline
\end{tabular}

\subsection{RTAD vs. Static Dissemination Schemes}

Static systems, unlike adaptive or dynamic systems, are systems that remain substantially unchanged through out time. Regarding vehicular environments, static broadcast schemes always use the same broadcast dissemination policy, without changing their mode of operation. Thus, they are not able to dynamically adapt their behavior to the specific features of the environment (e.g., different vehicle densities, or time-varying conditions).

In this section we compare the performance of our adaptive proposal against existing static dissemination schemes, such as the NJL and the eMDR schemes. Table 6 and Figure 11 show the average results obtained by each broadcast scheme in terms of: (i) $P_{I n f}$, the value that represents the efficiency of the dissemination process according the percentage of informed vehicles, and (ii) $M_{\text {recv }}$, the number of messages received by each vehicle, when varying the vehicle density and the city roadmap.

As shown, under low vehicle densities (i.e., 25 vehicles $/ \mathrm{km}^{2}$ ), the performance of RTAD is close to the results obtained by the most appropriate static broadcast scheme in sparse environments, i.e., the eMDR scheme. There are 


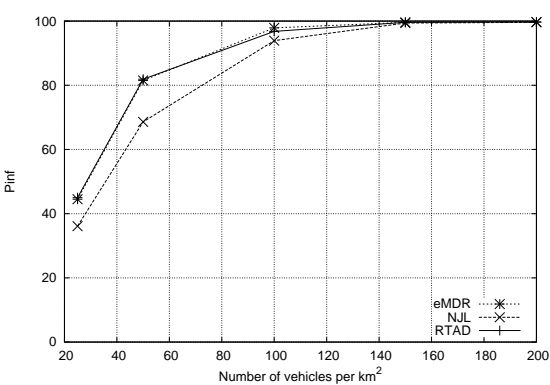

(a)

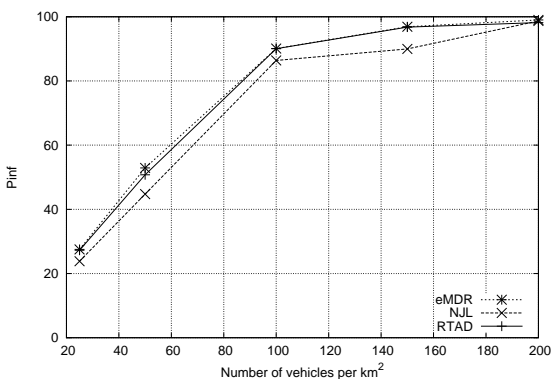

(b)

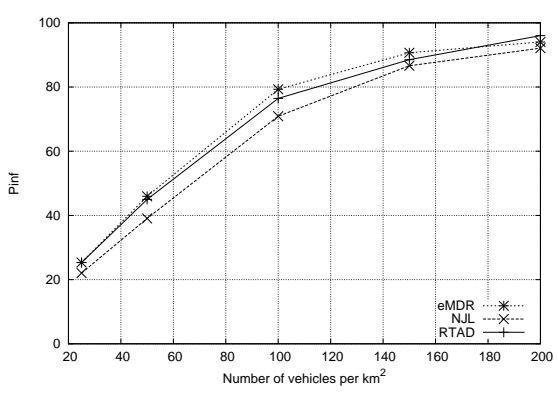

(c)

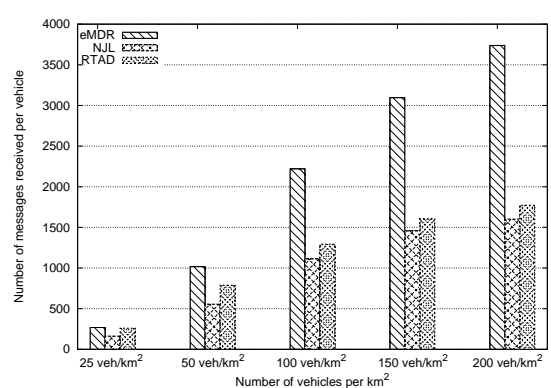

(d)

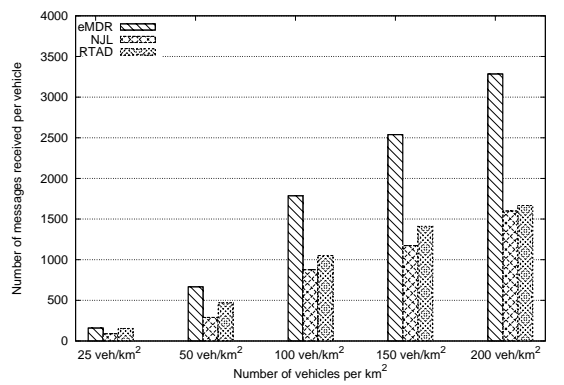

(e)

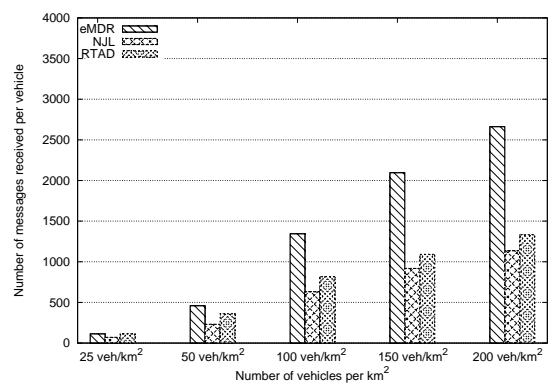

(f)

Figure 11: Informed vehicles $\left(P_{\text {Inf }}\right)$ and Messages received $\left(M_{r e c v}\right)$ when varying the vehicle density and the city roadmap: Los Angeles ((a) and (d)), Amsterdam ((b) and (e)), and Sydney ((c) and (f)). 
no significant changes in terms of informed vehicles, whereas there is a slight reduction in the number of messages received in all the tested scenarios when comparing the two schemes. The performance of the NJL scheme is worse under low vehicle densities; this becomes especially noticeable in the map of Los Angeles.

As the vehicle density increases, we see how the RTAD scheme still provides results very close to eMDR in terms of notified vehicles, although it only produces a fraction of the messages used by eMDR. The NJL scheme is the scheme that achieves the lowest value in the number of received messages; however, RTAD compensates the additional messages introduced by increasing the percentage of informed vehicles, which is clearly visible in the results obtained with the maps of Amsterdam and Sydney.

Finally, for the highest densities tested, RTAD is able to combine the best features of the two static schemes: the percentage of informed vehicles is very close to eMDR, but it generates an amount of messages comparable to those generated by NJL. Note that RTAD informs more vehicles than eMDR in the Sydney scenario under the highest density (200 vehicles $/ \mathrm{km}^{2}$ ), whereas the number of messages received is reduced by half. It is remarkable how the RTAD scheme provides optimal results in all situations compared to different static schemes, ranging from low densities with complex maps, to high densities in simple scenarios. By prioritizing the percentage of vehicles notified, and by reducing the number of messages as much as possible, it is able to reduce the channel overhead and optimize the message dissemination process.

\subsection{RTAD vs. Adaptive Dissemination Schemes}

Adaptive systems are able to respond to environmental changes, adapting its operation mode so as to face these changes in the best possible way. Regarding vehicular environments, adaptive schemes are able to adapt the dissemination policy to the specific conditions of the scenario, thereby improving the dissemination process, or reducing the channel contention. In this section we compare the performance of our proposal against other existing adaptive dis- 
semination schemes: DV-CAST (Tonguz et al., 2010), UV-CAST (Viriyasitavat et al., 2011), and FDPD (Sormani et al., 2006).

- Tonguz et al. (2010) presented the Distributed Vehicular Broad-CAST (DV-CAST) protocol. Specifically, DV-CAST is a distributed broadcast protocol that relies only on local topology information for handling broadcast messages in VANETs. DV-CAST handles the broadcast storm and the disconnected network problems simultaneously, while incurring a small amount of additional overhead. In particular, the DV-CAST protocol relies on local topology information (i.e., a list of one-hop neighbors) as the main criterion to determine how to handle message rebroadcasting, adapting the dissemination process depending on the density of neighbor vehicles, their position, and their direction. In our simulations we used the weighted p-persistence broadcast suppression technique, as it was the technique selected by authors (Tonguz et al., 2010).

- Viriyasitavat et al. (2011) proposed the Urban Vehicular broadCAST (UVCAST) protocol to reduce the broadcast storm problem while solving disconnected network problems in urban VANETs. The UV-CAST algorithm selects different mechanisms for message dissemination in VANETs, differentiating between well-connected and disconnected network regimes. Vehicles in well-connected regimes rebroadcast incoming messages after a wait time if no redundant messages are received. Vehicles under disconnected regimes must decide if they are suitable for the Store-CarryForward (SCF) task, forwarding the message whenever they meet new neighbors. The SCF task is assigned to vehicles that have a small expected time before they detect new neighbors, obtained as the boundary vehicles of the neighbors within communication range.

- Sormani et al. (2006) defined a message propagation function that encodes information about both target areas and preferred routes. Then, they showed how this function can be exploited in several routing proto- 
Table 7: Performance of the different adaptive dissemination systems when varying the vehicle density and the city roadmap.

\begin{tabular}{|c|c|c|c|c|c|c|c|}
\hline \multirow[b]{2}{*}{$\mathrm{v} / \mathrm{km}^{2}$} & \multirow[b]{2}{*}{ Bcast } & \multicolumn{2}{|c|}{ Los Angeles } & \multicolumn{2}{|c|}{ Amsterdam } & \multicolumn{2}{|c|}{ Sydney } \\
\hline & & $P_{I n f}$ & $M_{\text {recv }}$ & $P_{I n f}$ & $M_{\text {recv }}$ & $P_{I n f}$ & $M_{\text {recv }}$ \\
\hline \multirow{4}{*}{25} & RTAD & 54.30 & 261.43 & 37.33 & 154.08 & 33.47 & 112.55 \\
\hline & DV-CAST & $56.37(+2.07 \%)$ & $377.98(+44.6 \%)$ & $38.77(+1.43 \%)$ & $249.46(+61.9 \%)$ & $35.47(+2.00 \%)$ & $170.73(+51.7 \%)$ \\
\hline & FDPD & $34.67(-19.63 \%)$ & $58.64(-77.6 \%)$ & $24.60(-12.73 \%)$ & $45.67(-70.4 \%)$ & $22.33(-11.13 \%)$ & $33.51(.70 .23 \%)$ \\
\hline & UV-CAST & $32.83(-21.47 \%)$ & $50.17(-80.8 \%)$ & $23.10(-14.23 \%)$ & $39.88(-74.1 \%)$ & $23.33(-10.13 \%)$ & $35.17(-68.8 \%)$ \\
\hline \multirow{4}{*}{50} & RTAD & 86.33 & 789.70 & 61.00 & 470.20 & 55.07 & 360.68 \\
\hline & DV-CAST & $89.87(+3.53 \%)$ & $1414.48(+79.1 \%)$ & $64.57(+3.57 \%)$ & $1042.66(+121.7 \%)$ & $58.67(+3.60 \%)$ & $721.30(+100.0 \%)$ \\
\hline & FDPD & $59.20(-27.13 \%)$ & $200.84(-74.6 \%)$ & $39.50(-21.50 \%)$ & $128.53(-72.7 \%)$ & $37.37(-17.70 \%)$ & $98.09(-72.80 \%)$ \\
\hline & UV-CAST & $47.77(-38.57 \%)$ & $95.45(-87.9 \%)$ & $34.67(-26.33 \%)$ & $68.63(-85.4 \%)$ & $30.17(-24.90 \%)$ & $59.75(-83.4 \%)$ \\
\hline \multirow{4}{*}{100} & RTAD & 97.63 & 1294.83 & 92.53 & 1051.98 & 82.23 & 816.05 \\
\hline & DV-CAST & $98.50(+0.87 \%)$ & $2793.41(+115.7 \%)$ & $94.33(+1.80 \%)$ & $2462.22(+134.1 \%)$ & $85.57(+3.33 \%)$ & $1932.45(+136.8 \%)$ \\
\hline & FDPD & $85.20(-12.43 \%)$ & $748.76(-42.2 \%)$ & $60.27(-32.27 \%)$ & $457.05(-56.6 \%)$ & $55.50(-26.73 \%)$ & $357.96(-56.14 \%)$ \\
\hline & UV-CAST & $62.20(-35.43 \%)$ & $138.10(-89.3 \%)$ & $47.63(-44.90 \%)$ & $129.96(-87.6 \%)$ & $47.10(-35.13 \%)$ & $98.54(-87.9 \%)$ \\
\hline \multirow{4}{*}{150} & RTAD & 99.63 & 1605.60 & 97.53 & 1411.88 & 90.40 & 1092.46 \\
\hline & DV-CAST & $97.67(-1.97 \%)$ & $3488.61(+117.3 \%)$ & $97.83(+0.30 \%)$ & $3209.42(+127.3 \%)$ & $91.30(+0.90 \%)$ & $2725.15(+149.5 \%)$ \\
\hline & FDPD & $89.27(-10.37 \%)$ & $1286.34(-19.9 \%)$ & $71.50(-26.03 \%)$ & $798.94(-43.4 \%)$ & $70.07(-20.33 \%)$ & $685.54(-37.25 \%)$ \\
\hline & UV-CAST & $71.30(-28.33 \%)$ & $206.13(-87.2 \%)$ & $55.80(-41.73 \%)$ & $157.02(-88.9 \%)$ & $49.60(-40.80 \%)$ & $100.40(-90.8 \%)$ \\
\hline \multirow{4}{*}{200} & RTAD & 99.73 & 1773.84 & 98.57 & 1666.42 & 96.77 & 1331.97 \\
\hline & DV-CAST & $99.73(+0.00 \%)$ & $4044.44(+128.0 \%)$ & $98.63(+0.07 \%)$ & $3767.27(+126.1 \%)$ & $97.13(+0.37 \%)$ & $3358.09(+152.1 \%)$ \\
\hline & FDPD & $96.37(-3.37 \%)$ & $1853.97(+4.5 \%)$ & $83.67(-14.90 \%)$ & $1308.7(-21.5 \%)$ & $78.03(-18.73 \%)$ & $1021.07(-23.34 \%)$ \\
\hline & UV-CAST & $76.70(-23.03 \%)$ & $254.80(-85.6 \%)$ & $65.37(-33.20 \%)$ & $209.64(-87.4 \%)$ & $51.33(-45.43 \%)$ & $127.45(-90.4 \%)$ \\
\hline
\end{tabular}

cols. Specifically, they proposed the Function-Driven Probabilistic Diffusion (FDPD), a probabilistic message dissemination scheme that uses a propagation function calculated by means of the distance between sender and receiver, to determine the forwarding vehicles and reduce the broadcast storm problem.

Table 7 and Figure 12 show the results obtained by each adaptive broadcast system in terms of informed vehicles and number of messages received by vehicle, when varying the vehicle density and the city roadmap.

First of all, it is noteworthy how the UV-CAST scheme is extremely focused on the reduction of messages. It produces the lowest number of messages in all the tested scenarios, up to $90.8 \%$ fewer messages received when compared to RTAD. However, this massive reduction presents an important drawback, since it causes a very slow and inefficient dissemination process. Thus, the UV-CAST is an unsuitable scheme for warning message dissemination, where the main objective is to inform as many vehicles as soon as possible. However, 


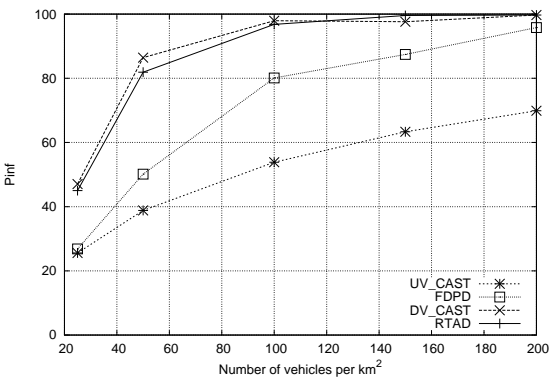

(a)

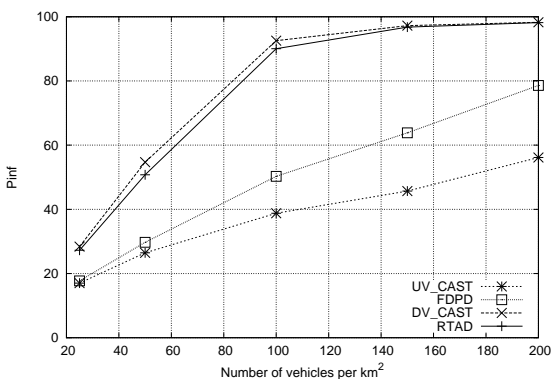

(b)

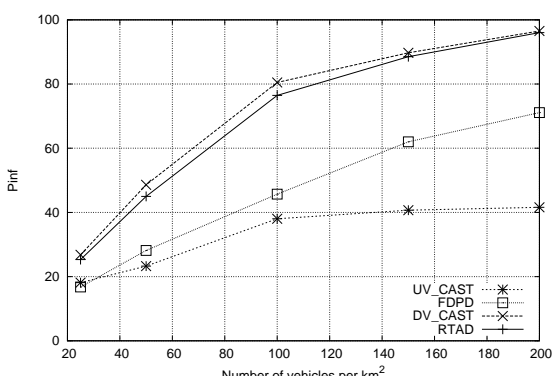

(c)

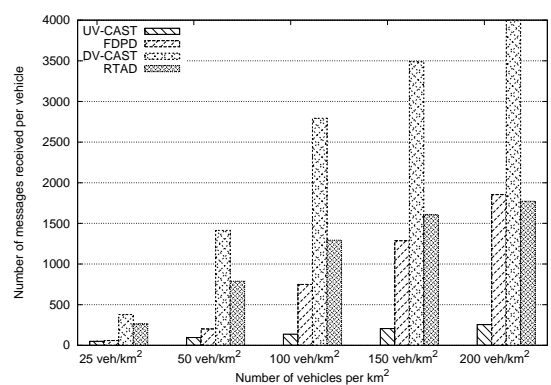

(d)

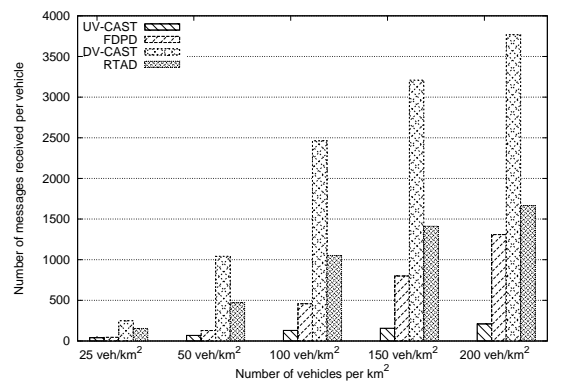

(e)

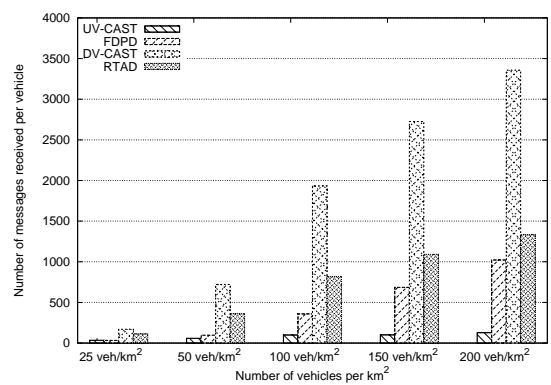

(f)

Figure 12: Informed vehicles $\left(P_{\text {Inf }}\right)$ and Messages received $\left(M_{r e c v}\right)$ when varying the vehicle density and the city roadmap: Los Angeles ((a) and (d)), Amsterdam ((b) and (e)), and Sydney ((c) and (f)). 
this scheme could be useful to disseminate non-critical information, such as advertisements, which do not present the delay requirements associated to traffic safety applications. The DV-CAST scheme obtains results close to the proposed RTAD scheme in terms of informed vehicles. In most of the tested situations the difference between the values obtained by both algorithms is less than $3 \%$, with situations favorable to DV-CAST, especially under low vehicle densities. However, in high vehicle densities, RTAD is able to inform almost the same number of vehicles while considerably reducing the number of messages received per vehicle. Focusing on the number of received messages, we can observe how RTAD is able to reduce the number of messages by more than half in most of the scenarios, especially when the vehicle density increases. This effect makes the RTAD algorithm more robust against broadcast storms, and thus more suitable for environments with a high density of vehicles. Such environments are more prone to increase the number of messages, thus producing channel contention and packet collisions.

Finally, the FDPD scheme offers intermediate results in terms of informed nodes. It is able to outperform UV-CAST, but it does not reach the values obtained by DV-CAST and RTAD. Regarding the number of messages, there is an exponential increment as vehicle density increases. For example, when simulating 25 veh. $/ \mathrm{km}^{2}$ in Los Angeles, the number of messages is similar to UV-CAST, whereas increasing the density to $200 \mathrm{veh} . / \mathrm{km}^{2}$, it offers values higher than RTAD.

To sum up, the proposed RTAD system is able to inform more vehicles than the UV-CAST algorithm in less time, while maintaining a low number of messages produced compared to DV-CAST. Hence, RTAD achieves an optimal balance between the two metrics (i.e., informed vehicles and messages received), making it suitable for a wide variety of scenarios. 


\section{Related Work}

In the networking literature we can find several works that present adaptive mechanisms specially designed to enhance message dissemination in vehicular communications. In this section we present some of the most representative works.

Sun et al. (2000) proposed two new broadcast protocols (namely TRADE and DDT), that make use of global positioning system (GPS) information to enhance the performance of broadcast service in vehicle-to-vehicle (V2V) communications. In particular, the two algorithms effectively reduce the number of re-broadcast messages without affecting the number of vehicles that receive the information. However, compared to our approach, these protocols are static (i.e., the dissemination policy does not change through out time), and they were mainly focused to be used in simple highway scenarios.

Ros et al. (2009) presented a broadcast protocol suitable for a wide range of vehicular scenarios and traffic conditions. The protocol employs local position information acquired via periodic beacon messages. Identifiers of circulated broadcast messages are added to beacons as piggybacked acknowledgments. When waiting timeout expires, vehicle retransmits if it has at least one neighbor which did not acknowledge circulated message with the last beacon, and sets a new waiting period. Despite its simplicity, the protocol provides high reliability and efficiency by means of a simulation-based performance evaluation.

Monteiro et al. (2013) simulated highway and urban VANET scenarios of different sizes and vehicle densities. They studied parameters such as the node degree distribution, the clustering coefficient and the average shortest path length, then they showed how to use this information to improve existing VANET protocols. As an illustrative example, it is shown that, by adding new mechanisms that make use of this information, the overhead of the urban vehicular broadcasting (UV-CAST) protocol could be reduced substantially with no significant performance degradation. 
Xue-wen et al. (2010) proposed the Transmission Range Adaptive Broadcast (TRAB), a broadcast algorithm for VANETs. By considering the transmission ranges of vehicles together with the inter-vehicle distances, TRAB calculates the waiting time to select the relay vehicles in accordance with the additional coverage area of adjacent vehicles to ensure that fewer relay vehicles will be used to forward the warning messages. However, this scheme is designed to obtain an efficient propagation of warning messages in highway scenarios alone, making it unsuitable for scenarios with complex topologies where the dissemination of warnings in all directions surrounding the critical area would be required.

Slavik et al. (2012) proposed the Rate-Adaptive Broadcast (RAB) protocol for information dissemination in VANETs. RAB adapts to the network conditions, although it does not require any knowledge of the network topology. By assuming a VANET dissemination application with fixed periodic updates, $\mathrm{RAB}$ is able to use a decision threshold control algorithm based on the message rate. If the new message rate dips below its long-run average, the decision threshold is adjusted to improve message propagation. Otherwise, RAB adjusts the decision threshold to keep the duplicate message rate within an efficient range. Thus, RAB jointly optimizes the broadcast message delivery rate and the bandwidth consumption. Unlike the TRAB scheme, the use of RAB is not restricted to highways; nevertheless, the roadmap layout is not used to select the vehicles to forward the messages. Instead, the scenario is modeled like a free space environment where vehicles only try to send messages as far away as possible, without accounting for the different blind areas that buildings may produce during the dissemination process.

Sommer et al. (2011) proposed the Adaptive Traffic Beacon (ATB), a fully distributed message dissemination protocol which uses adaptive beaconing based on two key metrics: message utility and channel quality. Authors showed that adaptive beaconing leads to a much broader dissemination of messages (in terms of penetration rate) than flooding-based approaches, although at a slower rate. The main objective of ATB is to exchange information in knowledge bases by sending beacons as frequently as possible, while maintaining a congestion-free 
wireless channel. However, their proposal cannot be applied in time-critical safety applications where the quick dissemination of warning messages is crucial. Additionally, authors only tested their proposal in a roadmap portion of Ingolstadt, Germany.

Ke et al. (2011) proposed the Adaptive Connectivity Data Dissemination Scheme (ACDDS), a data dissemination strategy where the vehicles calculate the network connectivity in their neighborhood by using the distributed vehicular density perception algorithm. A hop limit function is established on the basis of the Euclidean distance and the vehicular density between the vehicles and the hotspot. Simulation experiments show that the delivery ratio and the delay values for the proposed scheme are similar to the epidemic routing protocol, while reducing the number of message copies by $37.5 \%$. To validate their proposal, authors used real mobility traces obtained from 479 taxis in the San Francisco area. However, they did not use mobility traces for other types of vehicles, the density of vehicles was really low, and they did not account for the presence of obstacles in wireless signal propagation. These assumptions could lead to unrepresentative results.

Schwartz et al. (2012) proposed a data dissemination protocol for VANETs that distributes data utility fairly over vehicles while adaptively controlling the network load. The protocol relies only on local knowledge to achieve fairness using concepts of Nash Bargaining from game theory. Simulation results show that their algorithm presents a higher fairness index, while maintaining a high level of bandwidth utilization efficiency. In addition, the rate of transmissions is adaptively controlled as new information about the environment is collected. However, the vehicular density of the scenarios where their proposal was tested was very low (i.e., only 20 vehicles $/ \mathrm{km}^{2}$ ). Additionally, it is not clearly explained if their simulations accounted for the effect of obstacles in wireless signal propagation, and the benefits of their proposal in terms of vehicles informed.

Overall, we find that existing adaptive dissemination techniques for VANETs usually consider features related to vehicles in the scenario, such as their density, speed, and location, to adapt the performance of the dissemination process. 
However, most of the works in the literature are designed for highway scenarios where messages are only propagated in one direction, or focused on end-to-end routing. Additionally, most of them do not account for the effect of buildings and other obstacles during the dissemination of messages, which may lead to wrong conclusions. Hence, these approaches are not useful when attempting to warn the highest possible number of vehicles about dangerous situations in realistic vehicular environments, especially in urban environments.

\section{Conclusions}

In this paper we proposed RTAD, an adaptive warning message dissemination algorithm that selects the optimal broadcast scheme in a VANET scenario based on two different metrics: (i) the percentage of informed vehicles, a particularly determinant factor in warning message dissemination, and (ii) the number of messages received by each vehicle, an important factor which indicates the channel contention and the possibility of broadcast storms during the dissemination of alert messages.

In addition, we presented a new broadcast scheme called Nearest Junction Located (NJL), which was specially designed for scenarios presenting high vehicular densities or simple topologies, where broadcast storms are prone to occur. The NJL scheme is designed to reduce the number of messages received per vehicle without noticeably affecting the percentage of informed vehicles.

Experiments showed how our RTAD system is able to dynamically select the optimal dissemination scheme in all the scenarios, thereby adapting to the specific characteristics of them. Moreover, it outperforms static dissemination schemes as well as existing adaptive dissemination systems such as UV-CAST, FDPD, and DV-CAST. Our adaptive dissemination mechanism allows each vehicle to select the optimal broadcast scheme in real time, thus obtaining better results in terms of percentage of vehicles informed and significantly reducing the number of messages sent, while avoiding overloading the channel and improving the performance of other VANET applications. 


\section{Acknowledgments}

This work was partially supported by the Ministerio de Ciencia e Innovación, Spain, under Grant TIN2011-27543-C03-01, as well as the Government of Aragón and the European Social Fund (T91 Research Group).

\section{References}

Bi, Y., Cai, L., Shen, X., Zhao, H., May 2010. A Cross Layer Broadcast Protocol for Multihop Emergency Message Dissemination in Inter-Vehicle Communication. In: IEEE International Conference on Communications (ICC). pp. $1-5$.

Boukerche, A., Oliveira, H. A., Nakamura, E. F., Loureiro, A. A., 2008. Vehicular ad hoc networks: A new challenge for localization-based systems. Computer Communications 31 (12), 2838-2849.

Fall, K., Varadhan, K., February 2000. ns notes and documents. The VINT Project. UC Berkeley, LBL, USC/ISI, and Xerox PARC.

URL http://www.isi.edu/nsnam/ns/ns-documentation.html

Fogue, M., Garrido, P., Martinez, F. J., Cano, J.-C., Calafate, C. T., Manzoni, P., March 2012a. A Realistic Simulation Framework for Vehicular Networks. In: 5th International ICST Conference on Simulation Tools and Techniques (SIMUTools 2012), Desenzano, Italy. pp. 37-46.

Fogue, M., Garrido, P., Martinez, F. J., Cano, J.-C., Calafate, C. T., Manzoni, P., December 2012b. Evaluating the impact of a novel message dissemination scheme for vehicular networks using real maps. Transportation Research Part C: Emerging Technologies 25, 61-80.

Ghandour, A. J., Felice, M. D., Artail, H., Bononi, L., 2014. Dissemination of safety messages in IEEE $802.11 \mathrm{p} /$ wave vehicular network: Analytical study and protocol enhancements. Pervasive and Mobile Computing 11, 3-18. 
Jiang, D., Chen, Q., Delgrossi, L., 2008. Optimal data rate selection for vehicle safety communications. In: Proceedings of the Fifth ACM International Workshop on VehiculAr Inter-NETworking. VANET '08. ACM, New York, NY, USA, pp. 30-38.

Ke, W., Wei-dong, Y., Ji-Zhao, L., Dan-tuo, Z., 2011. An adaptive connectivity data dissemination scheme in vehicular ad-hoc networks. In: 7th Intl. Conference on Computational Intelligence and Security (CIS). pp. 531-535.

Krajzewicz, D., Erdmann, J., Behrisch, M., Bieker, L., December 2012. Recent development and applications of SUMO - Simulation of Urban MObility. International Journal On Advances in Systems and Measurements 5 (3\&4), $128-138$.

Krauss, S., Wagner, P., Gawron, C., 1997. Metastable states in a microscopic model of traffic flow. Physical Review E 55 (5), 5597-5602.

Kumar, N., Chilamkurti, N., Rodrigues, J. J., 2014. Learning automata-based opportunistic data aggregation and forwarding scheme for alert generation in vehicular ad hoc networks. Computer Communications 39, 22-32.

Li, X., Shu, W., Li, M., Luo, P.-E., Huang, H., Wu, M.-Y., 2008. Traffic data processing in vehicular sensor networks. In: 17th International Conference on Computer Communications and Networks (ICCCN'08). pp. 1-5.

Martinez, F. J., Cano, J.-C., Calafate, C. T., Manzoni, P., May 2008. Citymob: a mobility model pattern generator for VANETs. In: IEEE Vehicular Networks and Applications Workshop (Vehi-Mobi, held with ICC), Beijing, China.

Martinez, F. J., Fogue, M., Coll, M., Cano, J.-C., Calafate, C., Manzoni, P., 2010. Evaluating the impact of a novel warning message dissemination scheme for VANETs using real city maps. In: Crovella, M., Feeney, L., Rubenstein, D., Raghavan, S. (Eds.), NETWORKING 2010. Vol. 6091 of Lecture Notes in Computer Science. Springer Berlin / Heidelberg, pp. 265-276. 
Martinez, F. J., Fogue, M., Toh, C. K., Cano, J.-C., Calafate, C. T., Manzoni, P., 2013. Computer simulations of VANETs using realistic city topologies. Wireless Personal Communications 69 (2), 639-663.

Monteiro, R., Sargento, S., Viriyasitavat, W., Tonguz, O. K., 2013. Improving VANET protocols via network science. Computing Research Repository.

Noor, R., Khokhar, R., Jabbarpour, R., Khorsandroo, S., Khamis, N., Michael, O., 2012. Using VANET to support green vehicle communications for urban operation rescue. In: 12th International Conference on ITS Telecommunications (ITST). pp. 324-328.

OpenStreetMap, 2012. Collaborative project to create a free editable map of the world.

URL http://www .openstreetmap.org

Ros, F., Ruiz, P., Stojmenovic, I., 2009. Reliable and efficient broadcasting in vehicular ad hoc networks. In: IEEE 69th Vehicular Technology Conference (VTC Spring 2009). pp. 1-5.

Salvo, P., De Felice, M., Cuomo, F., Baiocchi, A., 2012. Infotainment traffic flow dissemination in an urban VANET. In: IEEE Global Communications Conference (GLOBECOM). pp. 67-72.

Sanchez-Medina, J., Galan-Moreno, M., Rubio-Royo, E., 2010. Traffic signal optimization in La Almozara district in Saragossa under congestion conditions, using genetic algorithms, traffic microsimulation, and cluster computing. IEEE Transactions on Intelligent Transportation Systems 11 (1), 132-141.

Sanguesa, J. A., Fogue, M., Garrido, P., Martinez, F. J., Cano, J.-C., Calafate, C. T., Manzoni, P., 2013a. An infrastructureless approach to estimate vehicular density in urban environments. Sensors 13 (2), 2399-2418.

Sanguesa, J. A., Fogue, M., Garrido, P., Martinez, F. J., Cano, J.-C., Calafate, C. T., Manzoni, P., 2013b. On the selection of optimal broadcast schemes 
in VANETs. In: Proceedings of the 16th ACM international conference on Modeling, analysis and simulation of wireless and mobile systems (MSWIM). ACM, New York, NY, USA, pp. 411-418.

Schwartz, R., Ohazulike, A., Sommer, C., Scholten, H., Dressler, F., Havinga, P., 2012. Fair and adaptive data dissemination for traffic information systems. In: IEEE Vehicular Networking Conference (VNC). pp. 1-8.

Slavik, M., Mahgoub, I., January 2010. Stochastic Broadcast for VANET. In: 7th IEEE Consumer Communications and Networking Conference (CCNC). Las Vegas, USA, pp. 1-5.

Slavik, M., Mahgoub, I., Alwakeel, M., 2012. Adapting statistical multi-hop wireless broadcast protocol decision thresholds using rate control. In: 9th International Conference on High Capacity Optical Networks and Enabling Technologies (HONET). pp. 32-36.

Sommer, C., Tonguz, O., Dressler, F., 2011. Traffic information systems: efficient message dissemination via adaptive beaconing. IEEE Communications Magazine 49 (5), 173-179.

Sormani, D., Turconi, G., Costa, P., Frey, D., Migliavacca, M., Mottola, L., 2006. Towards lightweight information dissemination in inter-vehicular networks. In: Proceedings of the 3rd international workshop on Vehicular ad hoc networks. VANET '06. ACM, New York, NY, USA, pp. 20-29.

Sun, M.-T., Feng, W.-C., Lai, T.-H., Yamada, K., Okada, H., Fujimura, K., September 2000. GPS-based message broadcast for adaptive inter-vehicle communications. In: 52nd IEEE Vehicular Technology Conference,VTC Fall. Vol. 6. pp. 2685-2692.

Suriyapaibonwattana, K., Pomavalai, C., October 2008. An effective safety alert broadcast algorithm for VANET. In: International Symposium on Communications and Information Technologies (ISCIT). pp. 247-250. 
Tonguz, O., Wisitpongphan, N., Bai, F., 2010. DV-CAST: A distributed vehicular broadcast protocol for vehicular ad hoc networks. IEEE Wireless Communications 17 (2), 47-57.

Tseng, Y.-C., Ni, S.-Y., Chen, Y.-S., Sheu, J.-P., 2002. The broadcast storm problem in a mobile ad hoc network. Wireless Networks 8, 153-167.

Viriyasitavat, W., Tonguz, O., Bai, F., Nov. 2011. UV-CAST: an urban vehicular broadcast protocol. IEEE Communications Magazine 49 (11), 116-124.

Wisitpongphan, N., Tonguz, O., Parikh, J., Mudalige, P., Bai, F., Sadekar, V., 2007. Broadcast storm mitigation techniques in vehicular ad hoc networks. IEEE Wireless Communications 14, 84-94.

Xue-wen, W., Wei, Y., Shi-ming, S., Hui-bin, W., 2010. A transmission range adaptive broadcast algorithm for vehicular ad hoc networks. In: Proceedings of the 2010 Second International Conference on Networks Security, Wireless Communications and Trusted Computing - Volume 01. NSWCTC '10. IEEE Computer Society, Washington, DC, USA, pp. 28-32.

Zhou, L., Zheng, B., Geller, B., Wei, A., Xu, S., Li, Y., 2008. Cross-layer rate control, medium access control and routing design in cooperative VANET. Computer Communications 31 (12), 2870-2882.

ZunZun, 2012. Online Curve Fitting and Surface Fitting Web Site. URL http://www.zunzun.com 